This item was submitted to Loughborough's Research Repository by the author.

Items in Figshare are protected by copyright, with all rights reserved, unless otherwise indicated.

\title{
A universal framework for modelling measured velocity in laser vibrometry with applications
}

PLEASE CITE THE PUBLISHED VERSION

http://dx.doi.org/10.1016/j.ymssp.2011.06.022

PUBLISHER

(C) Elsevier Ltd.

VERSION

AM (Accepted Manuscript)

LICENCE

CC BY-NC-ND 4.0

\section{REPOSITORY RECORD}

Rothberg, Steve, and Mario Tirabassi. 2019. "A Universal Framework for Modelling Measured Velocity in Laser Vibrometry with Applications”. figshare. https://hdl.handle.net/2134/8881. 
This item was submitted to Loughborough's Institutional Repository (https://dspace.lboro.ac.uk/) by the author and is made available under the following Creative Commons Licence conditions.

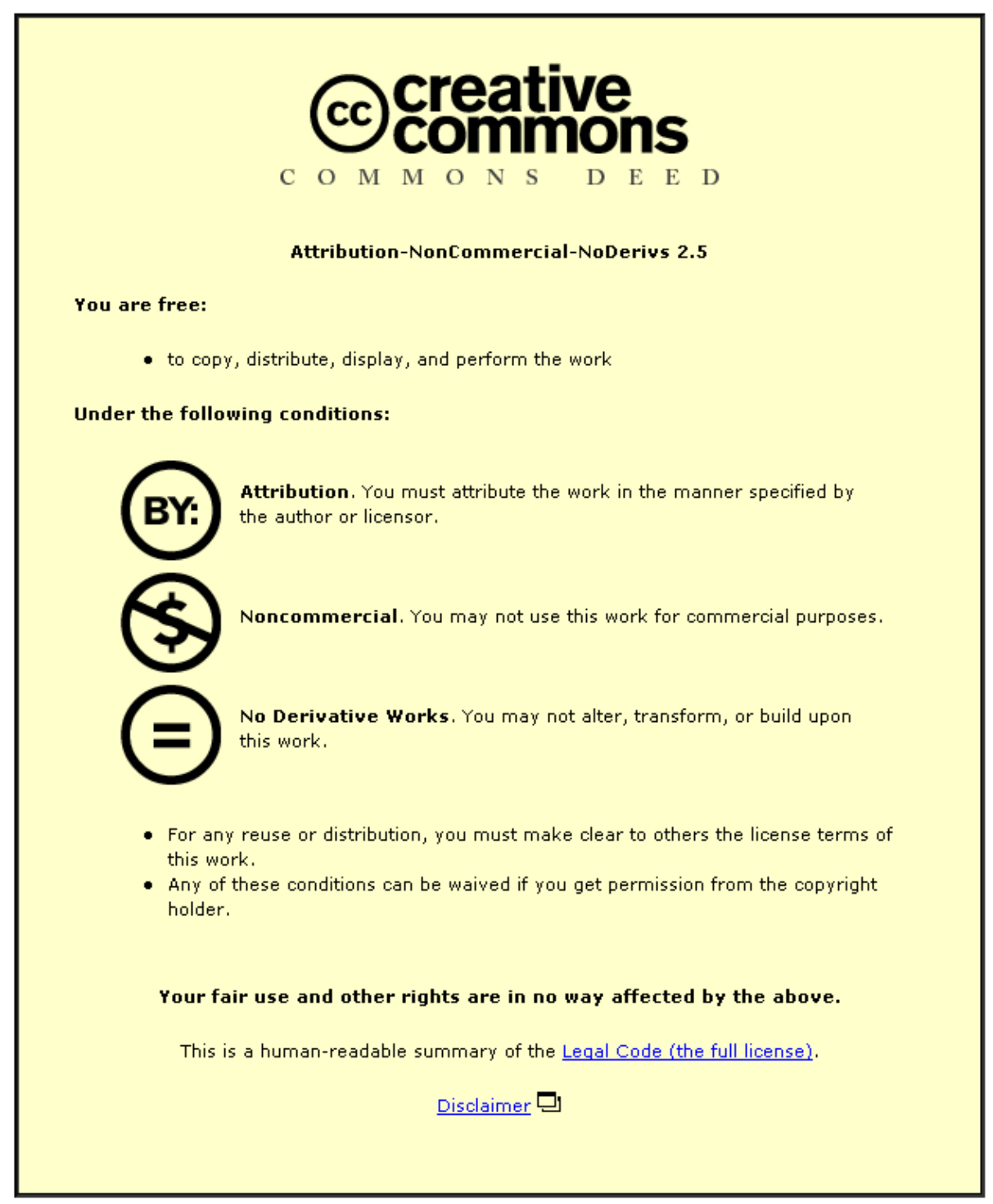

For the full text of this licence, please go to: http://creativecommons.org/licenses/by-nc-nd/2.5/ 


\section{A Universal Framework for Modelling Measured Velocity in Laser Vibrometry with Applications}

Steve J. Rothberg and Mario Tirabassi

Wolfson School of Mechanical and Manufacturing Engineering

Loughborough University, Loughborough, Leicestershire, LE11 3TU, UK.

Corresponding author: Steve Rothberg

e-mail: s.j.rothberg@lboro.ac.uk,

tel: +44 (0) 1509 227524,

fax: +44 (0) 1509227502 


\title{
A Universal Framework for Modelling Measured Velocity in Laser Vibrometry with Applications
}

\author{
Steve J. Rothberg and Mario Tirabassi \\ Wolfson School of Mechanical and Manufacturing Engineering \\ Loughborough University, UK
}

\begin{abstract}
:
This paper presents a novel, universally applicable framework for modelling measured velocity in laser vibrometry systems. The framework is introduced generically before demonstration of its application to three scanning vibrometer systems, each configured to measure vibration of a tracked point on a rotating target. The novelty in this vectorial framework lies in the combination of its elements which include vector descriptions of target velocity, optical device velocity at deflection points, laser beam orientations, incorporating reflection and refraction, and surface normals. Initial alignment and a full set of inevitable misalignments are incorporated by the modification of position vectors and the use of rotation matrices. Inclusion of components of measured velocity associated with moving optical devices is an important feature of the framework. The models derived and their validation against published data demonstrate how this versatile framework can be applied to any optical configuration measuring target motions with any level of complexity. The individual models are explored extensively and quantitatively through simulation. Small but inevitable misalignments are shown to generate measurable low order velocity components and their effects on the sensitivities to in-plane and out-of-plane components of target vibration are quantified.
\end{abstract}

KEYWORDS: Laser vibrometry, vibration measurement, scanning, tracking, misalignment.

\section{Introduction}

The laser Doppler vibrometer (LDV) is now well established as an effective alternative to traditional contacting vibration transducers. LDVs are technically well suited to general application but offer special benefits where measurement constraints are imposed either by the context, which may demand high frequency operation, high spatial resolution or remote transducer operation, or by the structure itself, which may be hot, light or rotating.

For contacting transducers, measurement axes are generally fixed by the surface orientation at the point of attachment. LDVs, however, measure velocity in the direction of the incident beam so the user has much greater freedom to orient the probe laser beam to select a desired vibration component (limited only by low collected light intensity). Both the direction of the laser beam and its incident point can be manipulated with an ease that cannot be matched by contacting transducers. The potential for automation of this relocation using optical devices (typically a pair of orthogonally 
mounted galvanometer mirrors) to scan point-by-point across a structure was recognised at an early stage [1]. Particular applications emerged in the automotive [2-4] and aerospace [5] industries, and today's scanning LDV (SLDV) state-of-the-art [6] offers automated, tri-axial vibration surveys on large, three-dimensional structures (such as a vehicle) using three SLDVs each mounted on a robot arm.

It is also possible to configure a SLDV to function in a continuous scanning mode by driving the beam deflection mirrors with continuous time varying signals, initially enabling extraction of particular vibration components [7] and ultimately enabling the target velocity profile along a predetermined path to be determined in a single measurement. In this latter case, post-processing of the measured velocity results in a series of coefficients that describe the operational deflection shape or the mode shape [8-13]. With the scan frequency synchronised with the target motion frequency [14], a tracking LDV measurement is performed in which the probe laser beam remains fixed on a particular point on the target. Tracking measurements have been performed on a number of rotor applications [15-18], on belts [19] (partial track) and on targets with oscillating parts fixed to a component with a large whole body motion such as windscreen wipers [20]. On rotating structures, the attractions of tracking and scanning simultaneously have also been explored [21-23].

Long before the introduction of tracking techniques, measurements directly from rotating targets (using stationary laser beams) had been proposed as an important LDV application as a result of noncontact operation and inherent immunity to shaft run-out (deviation from a perfectly circular crosssection). Indeed, one of the first LDV applications was for axial vibration measurement directly from a rotating turbine blade [24] and interest in this application continues [25-27]. A parallel beam instrument for angular vibration measurements has also been used in rotor applications, for example for assessment of torsional damper health [28], on rotating machines [29] and on railway wheel sets [30]. Attempts to perform radial vibration measurements on rotors, however, encountered a crosssensitivity to the radial component perpendicular to the radial component it is intended to measure as a consequence of oscillation in the position of the rotor centre relative to the fixed line of incidence of the beam [31]. The finding prompted development of a model to predict measured velocity for arbitrary beam orientation and arbitrary target motion $[32,33]$ resulting in confirmation that the crosssensitivity could not be resolved by laser beam orientation or manipulation. A resolution procedure based on simultaneous measurements was subsequently formulated [34].

More generally, however, all these experiences highlight the need to be able to model measured velocity in all cases where freedom is available over laser beam orientation, from basic tripod mounting to more advanced continuous scanning and tracking applications, and where target motions are complex. A first attempt to adapt the model for radial vibration measurements on rotors (using 
stationary laser beams) to axial vibration measurements on rotors using a scanning laser beam was successful [35] but the new model was not readily applicable to other scanning systems [e.g. 15, 17, $18,36]$. In addition, in recent times, greater attention has fallen on the effects of instrument misalignments with the aims of minimising uncertainty and optimising data interpretation. Existing models have incorporated a limited set of misalignments but the modelling methods are insufficiently flexible to incorporate conveniently a full set of misalignments for all optical devices in any system.

The proposed framework addresses this deficiency, demonstrating its versatility and universal applicability through application to three different systems: the long established dual mirror SLDV, the recently developed Dove prism SLDV [18] and the self-tracking LDV [17]. Application to tracking measurements on rotating components is chosen as it represents a significant challenge in modelling measured velocity but the SLDV models are built up in the most general sense possible with adaptation to tracking shown as one possible example of implementation. Structured in this way, the paper takes the reader through systems with increasing complexity including reflections at plane and conical surfaces and refractions. The models themselves are also part of the novelty in this paper. The dual mirror SLDV has been modelled previously $[14,35,37]$ but not in the detail or with the ease presented here. To the authors' knowledge, models of the Dove prism and self-tracking systems have not previously been published.

Importantly, this paper also acknowledges how measured velocity is affected by Doppler shifts occurring at deflections by moving optical devices. This principle has long been recognised and, in early LDVs, was exploited to frequency-shift the reference beam using either a rotating diffraction grating [38] or a Bragg cell [39]. (Today, the Bragg cell, effectively a moving diffraction grating, is the most common frequency shifting device in use). For moving optical devices in scanning heads, this previously neglected but important aspect is straightforwardly included in the framework.

The models presented might serve many purposes including performance verification for a new system, optimisation of system or component geometries, analyses of artefacts observed in scan paths and measured velocity, quantification of sensitivity to intended and unintended vibration components and investigation of the effects of inevitable misalignments. In the absence of misalignments, the equations derived could be manipulated to produce final expressions for scan path and total measured velocity. With misalignments, the algebraic effort increases significantly and the models are best implemented in software such as MATLAB. This latter approach has been taken in this paper. Typical instrument output spectra in the presence of misalignments and target vibrations are shown and then the effects of misalignments are studied in greater detail through simulation of a very large number of misaligned configurations. Model validation is undertaken wherever published data is available. 


\section{Elements of the universal framework}

The principal aim of the framework is the prediction of measured velocity but beam path, including scan path for scanning and tracking applications, is also predicted. While the interest in measured velocity is quite specific to LDV systems, the prediction of beam path can immediately be applied to any optical system. In this section, the key elements of the framework will be presented in a generic manner, outlining the systematic approach that is comprehensive, without simplification and unique in its universal applicability. The novelty of the framework lies in the combination of these elements.

\subsection{Surface velocity and measured velocity}

The fundamental relationship between measured velocity, $U_{m}$, and surface velocity at a point $P^{\prime}, \overrightarrow{V_{P^{\prime}}}$, is captured in the expression [40]:

$U_{m}=\frac{1}{2}\left(\hat{b}_{n+1}-\hat{b}_{n}\right) \cdot \overrightarrow{V_{P^{\prime}}}$

in which $\hat{b}_{n+1}$ and $\hat{b}_{n}$ are, respectively, unit vectors for the laser beam directions immediately after and immediately before the point $P^{\prime}$. Equation (1) can be applied to incidence at a target surface and to deflections at optical devices. For scattering from the target with light collected in direct backscatter, $\hat{b}_{n+1}=-\hat{b}_{n}$ and equation (1) simplifies to:

$U_{m}=-\hat{b}_{n} \cdot \overrightarrow{V_{P^{\prime}}}$

With light collected in direct backscatter, deflections at optical devices occur at the same point in both the outgoing and incoming beam path. Based on equation (1), the measured velocity associated with the double pass through a deflection point $P^{\prime}$ on an optical device is therefore given by:

$U_{m}=\frac{1}{2}\left(\hat{b}_{n+1}-\hat{b}_{n}\right) \cdot \overrightarrow{V_{P^{\prime}}}+\frac{1}{2}\left(-\hat{b}_{n}--\hat{b}_{n+1}\right) \cdot \overrightarrow{V_{P^{\prime}}}=\left(\hat{b}_{n+1}-\hat{b}_{n}\right) \cdot \overrightarrow{V_{P^{\prime}}}$

where the unit vector subscripts refer to the outgoing beam path. The total measured velocity is given by the sum of the individual velocities associated with the Doppler shifts at deflections at optical devices and at the target.

Equations (2a\&b) highlight the attraction of vector descriptions for surface velocity and beam orientation. This is facilitated by definition of a global $x y z$ coordinate system with origin $O$ fixed in space and unit vectors $\hat{x}, \hat{y}$ and $\hat{z}$ associated with its axes. For the applications in this paper, $O$ coincides with a convenient reference point on the target rotation axis in the absence of any vibration. The $z$-axis is aligned with the target rotation axis, again in the absence of vibration. With vibration, 
this target reference point moves to $O^{*}$ as a consequence of its velocity $\overrightarrow{V_{O^{*}}}$. Surface velocity at the incident point $P^{\prime}$ is then conveniently written in terms of the sum of the velocity of the reference point and the velocity of $P^{\prime}$ relative to $O^{*}$ as a result of rotation at angular velocity $\vec{\omega}$ about an instantaneous rotation axis passing through $O^{*}$ :

$\overrightarrow{V_{P^{\prime}}}=\overrightarrow{V_{O^{*}}}+\left(\vec{\omega} \times \overrightarrow{r_{P^{\prime}} / O^{*}}\right)$

where $\overrightarrow{r_{P^{\prime} / O^{*}}}$ is the position vector for $P^{\prime}$ relative to $O^{*} . \vec{\omega}$ includes both continuous target rotation and angular oscillations around the three coordinate axes. For vibrating targets, $P^{\prime}$ can change continuously and can also be affected by target shape but, for an illuminated target element considered to be rigid, equation (2a) can be expanded in terms of any known point along the line of the laser beam [32], described by the position vector $\overrightarrow{r_{0}}$ :

$U_{m}=-\hat{b}_{n} \cdot \overrightarrow{V_{P^{\prime}}}=-\hat{b}_{n} \cdot\left(\overrightarrow{V_{O^{*}}}+\vec{\omega} \times\left[\overrightarrow{r_{0}}-\overrightarrow{O O^{*}}\right]\right)$

where $\overrightarrow{O O^{*}}$ is the target displacement vector associated with the velocity $\overrightarrow{V_{O^{*}}}$. For applications such as the tracking of a bladed disk, the relative vibration velocity associated with target flexibility must be added to the rigid element velocity [35]. This must be written to accommodate time-dependency in the illuminated point, modifying equation (4a) to become:

$U_{m}=-\hat{b}_{n} \cdot \overrightarrow{V_{P^{\prime}(t)}}=-\hat{b}_{n} \cdot\left(\overrightarrow{V_{O^{*}}}+\vec{\omega} \times\left[\overrightarrow{r_{0}}-\overrightarrow{O O^{*}}\right]+\overrightarrow{V_{f}\left(P^{\prime}(t)\right)}\right)$

where $\overrightarrow{V_{f}\left(P^{\prime}(t)\right)}$ is the vector velocity at point $P^{\prime}(t)$ associated with target flexibility. For each individual model, analysis incorporating all motions is readily made using equation 4(b) and expansion of its vibration terms is given in Appendix A. To demonstrate the merits of this approach without over-complication, later simulations restrict target motions to an in-plane $(x)$ whole body vibration, $V_{O^{*} x} \hat{x}$, and an out-of plane $(z)$ flexible vibration, $V_{f z}\left(P^{\prime}(t)\right) \hat{z}$, combined with target rotation at $\Omega_{\mathrm{T}}$ around $\hat{z}$. This represents the important case of a rotating bladed disc attached to a whirling shaft for which the measured velocity, in terms of point $T^{\prime}$ along the beam, is written:

$U_{m}=-\hat{b}_{n} \cdot\left(V_{O^{*} x} \hat{x}+\Omega_{\mathrm{T}} \hat{z} \times\left[\overrightarrow{O T^{\prime}}-\overrightarrow{O O^{*}}\right]+V_{f z}\left(P^{\prime}(t)\right) \hat{z}\right)$

$T^{\prime}$ is the point where the laser beam intersects the $x y$ plane in which $O$ is located. To all intents and purposes, this is the plane of the target and $T^{\prime}$ is the illuminated point on the target. Strictly, target shape and vibration can move the illuminated point away from this plane but practically any difference will be small. This fine distinction over the location of $T^{\prime}$ does not affect the prediction of measured velocity which requires only a known point along the beam as shown by equation (4a). 
In applying the same principles to an optical device, a particular labelling convention is adopted. Without misalignment, a significant point such as a reference point on a rotation axis might be identified as $P$. With misalignments added, the new position of this point will be labelled $P^{*}$ and point $P$ may no longer lie on a surface of the optical device. On the surface containing $P^{*}$, the point through which the laser beam actually passes will be labelled $P^{\prime}$. Vectors $\overrightarrow{O P}, \overrightarrow{O P^{*}}$ and $\overrightarrow{O P^{\prime}}$ define these positions while, for example, the vector $\overrightarrow{P^{*} P^{\prime}}$ defines the path from $P^{*}$ to $P^{\prime} \cdot \overrightarrow{O P}$ and $\overrightarrow{O P^{*}}$ will be inputs to the models while $\overrightarrow{O P^{\prime}}$ and $\overrightarrow{P^{*} P^{\prime}}$ will be found as part of the model. Adopting this convention and combining equations (2b) and (3), the measured velocity associated with deflection at an optical device can be written:

$U_{m}=\left(\hat{b}_{n+1}-\hat{b}_{n}\right) \cdot\left(\overrightarrow{V_{P^{*}}}+\vec{\omega} \times \overrightarrow{P^{*} P^{\prime}}\right)$

If $P^{*}$ is a point on the device rotation axis, $\overrightarrow{V_{P^{*}}}$ will usually be zero, simplifying equation (5).

Exceptions include modelling the effects of vibrations of the device itself and attachment of a mirror to the target in a self-tracking LDV system.

\subsection{Beam orientation and surface normals}

Following the conventions shown in figure 1, unit vectors for beam orientation after each deflection are derived using vector expressions for, respectively, reflection and refraction [41]:

$\hat{b}_{n+1}=\hat{b}_{n}-2\left(\hat{b}_{n} \cdot \hat{n}_{P^{\prime}}\right) \hat{n}_{P^{\prime}}$

$\hat{b}_{n+1}=\left(\hat{b}_{n}-\left(\hat{b}_{n} \cdot \hat{n}_{P^{\prime}}\right) \hat{n}_{P^{\prime}}\right) \frac{\varepsilon_{n}}{\varepsilon_{n+1}}-\left(\sqrt{1-\left(\frac{\varepsilon_{n}}{\varepsilon_{n+1}}\right)^{2}\left(1-\left(\hat{b}_{n} \cdot \hat{n}_{P^{\prime}}\right)^{2}\right)}\right) \hat{n}_{P^{\prime}}$

where $\varepsilon_{n}$ and $\varepsilon_{n+1}$ are the refractive indeces for the media associated with beam directions $\hat{b}_{n}$ and $\hat{b}_{n+1}$ respectively. For both cases, the final beam orientation, $\hat{b}_{n+1}$, is related to the initial orientation, $\hat{b}_{n}$, through $\hat{n}_{P^{\prime}}$, the surface normal unit vector at the point $P^{\prime}$. Surface normals are also essential to location of deflection points and this points to the next key element in this framework.

\subsection{Rotation matrices}

Rotation matrices enable convenient formulation of vector expressions for initial beam orientation, surface normal, rotation axes of optical devices and coordinate transformations. Correct orientation is achieved through multiplication of a convenient initial orientation vector by rotation matrices which can be applied singly or in series to modify that initial vector. The matrices are dependent on the axis 
around which the rotation is performed. For rotations $\alpha, \beta$ and $\gamma$ around, respectively, $x$-, $y$ - and $z$ axes, these matrices are [42]:

$$
\begin{aligned}
& {[x, \alpha]=\left[\begin{array}{ccc}
1 & 0 & 0 \\
0 & \cos \alpha & -\sin \alpha \\
0 & \sin \alpha & \cos \alpha
\end{array}\right]} \\
& {[y, \beta]=\left[\begin{array}{ccc}
\cos \beta & 0 & \sin \beta \\
0 & 1 & 0 \\
-\sin \beta & 0 & \cos \beta
\end{array}\right]} \\
& {[z, \gamma]=\left[\begin{array}{ccc}
\cos \gamma & -\sin \gamma & 0 \\
\sin \gamma & \cos \gamma & 0 \\
0 & 0 & 1
\end{array}\right]}
\end{aligned}
$$

For example, to express surface normal of a rotating prism with a sloping face, an initial orientation in the $z$-direction might be modified by a rotation around $x$ for surface slope $\psi$ followed by a whole body rotation of the prism around $z$ by $\gamma$ :

$\hat{n}_{P^{*}}=\left[\begin{array}{lll}\hat{x} & \hat{y} & \hat{z}\end{array}\right][z, \gamma][x, \psi][0 \quad 0 \quad 1]^{T}$

Any convenient initial orientation can be chosen with an appropriate choice of a constant component in $\gamma$ then used to position the optical device at any desired starting angular position. For optical devices, the reflecting and refracting surfaces are often plane and so the surface normal at the reference point can be used for all points on that surface:

$\hat{n}_{P^{\prime}}=\hat{n}_{P^{*}}$

\subsection{Deflection points at optical devices}

Identification of deflection points defines beam path and enables derivation of the surface velocities that lead to measured velocity according to equation (5). Key points of incidence are found sequentially beginning from the position of the laser source, which is another input to the models. In each case, a system of three equations is solved; the first is a vector triangle relating the unknown point of incidence to the previous deflection point (or laser source position) through knowledge of beam orientation, the second is a vector triangle relating the unknown point of incidence to a known point on the same optical surface and the third is a dot product between the surface normal and a vector in the plane of the surface.

\subsection{Incorporating misalignments}


Finally, the framework must accommodate translational and angular misalignments throughout a system. Even when an instrument's optical head is carefully aligned by the manufacturer, small misalignments will remain and a significant source of misalignment will always be between the optical head and the target. Translational misalignments are accounted for in the position vectors of the known points on each surface. For example:

$\overrightarrow{O P^{*}}=\overrightarrow{O P}+\left[\begin{array}{lll}\hat{x} & \hat{y} & \hat{z}\end{array}\right]\left[\begin{array}{lll}\Delta x_{P} & \Delta y_{P} & \Delta z_{P}\end{array}\right]^{T}$

where $\left[\begin{array}{ccc}\Delta x_{P} & \Delta y_{P} & \Delta z_{P}\end{array}\right]^{T}$ is a column matrix containing the three components of this translational misalignment. Rotation matrices are used to incorporate angular misalignments into expressions for initial beam direction, each surface normal and the rotation axis vectors for optical devices. For example, incorporating angular misalignments of $\beta$ and $\alpha$ around $y$-and $x$-axes, respectively, into the surface normal in equation (8) gives:

$\hat{n}_{P^{*}}=\left[\begin{array}{lll}\hat{x} & \hat{y} & \hat{z}\end{array}\right][x, \alpha][y, \beta][z, \gamma][x, \psi][0 \quad 0 \quad 1]^{T}$

Typical translational and angular misalignment values are treated as inputs to the models. This paper considers only constant misalignments but time-varying misalignments, such as might be caused by vibrations of the optical devices, can be incorporated in exactly the same way.

\section{Dual mirror SLDV}

The dual mirror SLDV controls beam orientation using a pair of orthogonal mirrors. Without misalignments, it is possible to define the points $A, B$ and $C$ which correspond respectively to the nominal position of the laser source, a reference point on the rotation axis of the first mirror and a reference point on the rotation axis of the second mirror. In this analysis, the obvious points on the mirrors to select are those where a perfectly aligned laser beam would be incident if the first mirror is in a position to reflect the incident beam through $90^{\circ}$. With the geometry shown in figure $2 \mathrm{a}$, these points can be written as:

$$
\begin{aligned}
& \overrightarrow{O A}=\left[\begin{array}{lll}
\hat{x} & \hat{y} & \hat{z}
\end{array}\right]\left[\begin{array}{lll}
x_{A} & -d_{S} & z_{A}
\end{array}\right]^{T} \\
& \overrightarrow{O B}=\left[\begin{array}{lll}
\hat{x} & \hat{y} & \hat{z}
\end{array}\right]\left[\begin{array}{lll}
0 & -d_{S} & z_{A}
\end{array}\right]^{T} \\
& \overrightarrow{O C}=\left[\begin{array}{lll}
\hat{x} & \hat{y} & \hat{z}
\end{array}\right]\left[\begin{array}{lll}
0 & 0 & z_{A}
\end{array}\right]^{T}
\end{aligned}
$$

With translational misalignments, these points are modified as follows: 
$\overrightarrow{O A^{\prime}}=\overrightarrow{O A}+\left[\begin{array}{lll}\hat{x} & \hat{y} & \hat{z}\end{array}\right]\left[\begin{array}{lll}\Delta x_{A} & \Delta y_{A} & \Delta z_{A}\end{array}\right]^{T}$

$\overrightarrow{O B^{*}}=\overrightarrow{O B}+\left[\begin{array}{lll}\hat{x} & \hat{y} & \hat{z}\end{array}\right]\left[\begin{array}{lll}\Delta x_{B} & \Delta y_{B} & \Delta z_{B}\end{array}\right]^{T}$

$\overrightarrow{O C^{*}}=\overrightarrow{O C}+\left[\begin{array}{lll}\hat{x} & \hat{y} & \hat{z}\end{array}\right]\left[\begin{array}{lll}\Delta x_{C} & \Delta y_{C} & \Delta z_{C}\end{array}\right]^{T}$

Noting that the $x$-position of point $A$ is nominal, it is reasonable to set $\Delta x_{A}=0$. These points are shown in Figure $2 b$, together with the original mirror reference points. The surface normal for the first mirror, $\hat{n}_{B^{*}}$, can be written in terms of an initial alignment in the positive $x$-direction modified by a rotation matrix incorporating both mean and oscillatory components of angular position around the $z$ axis, combined as $\gamma_{1}$. This is further modified by angular misalignments $\beta_{1}$ and $\alpha_{1}$ around $y$-and then $x$-directions.

$\hat{n}_{B^{*}}=\left[\begin{array}{lll}\hat{x} & \hat{y} & \hat{z}\end{array}\right]\left[x, \alpha_{1}\right]\left[y, \beta_{1}\right]\left[z, \gamma_{1}\right]\left[\begin{array}{lll}1 & 0 & 0\end{array}\right]^{T}$

These misalignments also affect the rotation axis of this mirror, such that its unit vector, $\hat{z}_{1}$, deviates from $\hat{z}$ as follows:

$\hat{z}_{1}=\left[\begin{array}{lll}\hat{x} & \hat{y} & \hat{z}\end{array}\right]\left[x, \alpha_{1}\right]\left[y, \beta_{1}\right]\left[\begin{array}{lll}0 & 0 & 1\end{array}\right]^{T}$

The surface normal for the second mirror, $\hat{n}_{C^{*}}$, can be written in terms of an initial alignment in the negative $y$-direction modified by a rotation matrix incorporating both mean and oscillatory components of angular position around the $x$-axis, combined as $\alpha_{2}$. This is further modified by angular misalignments $\gamma_{2}$ and $\beta_{2}$ around $z$-and then $y$-directions with a similar effect on the unit vector for the mirror rotation axis, $\hat{x}_{2}$.

$$
\begin{aligned}
& \hat{n}_{C^{*}}=\left[\begin{array}{lll}
\hat{x} & \hat{y} & \hat{z}
\end{array}\right]\left[y, \beta_{2}\right]\left[z, \gamma_{2}\right]\left[x, \alpha_{2}\right]\left[\begin{array}{lll}
0 & -1 & 0
\end{array}\right]^{T} \\
& \hat{x}_{2}=\left[\begin{array}{lll}
\hat{x} & \hat{y} & \hat{z}
\end{array}\right]\left[y, \beta_{2}\right]\left[z, \gamma_{2}\right]\left[\begin{array}{lll}
1 & 0 & 0
\end{array}\right]^{T}
\end{aligned}
$$

With an initial laser beam orientation in the negative $x$-direction modified by angular misalignments $\gamma_{L}$ and $\beta_{L}$ around $z$ - and then $y$-directions, the expression for the initial beam orientation, $\hat{b}_{1}$, is found.

$\hat{b}_{1}=\left[\begin{array}{lll}\hat{x} & \hat{y} & \hat{z}\end{array}\right]\left[y, \beta_{L}\right]\left[z, \gamma_{L}\right][-1 \quad 0 \quad 0]^{T}$

Equation (5a) then provides the beam orientations, $\hat{b}_{2}$ and $\hat{b}_{3}$, after the first and second reflections respectively:

$\hat{b}_{2}=\hat{b}_{1}-2\left(\hat{b}_{1} \cdot \hat{n}_{B^{*}}\right) \hat{n}_{B^{*}}$ 
$\hat{b}_{3}=\hat{b}_{2}-2\left(\hat{b}_{2} \cdot \hat{n}_{C^{*}}\right) \hat{n}_{C^{*}}$

As set out in section 2.4, the position of incidence point $B^{\prime}$ is obtained from the following set of equations:

$\overrightarrow{O B^{\prime}}=\overrightarrow{O A^{\prime}}+\left|\overrightarrow{A^{\prime} B^{\prime}}\right| \hat{b}_{1}$

$\overrightarrow{O B^{\prime}}=\overrightarrow{O B^{*}}+\overrightarrow{B^{*} B^{\prime}}$

$\overrightarrow{B^{*} B^{\prime}} \cdot \hat{n}_{B^{*}}=0$

leading to:

$\overrightarrow{O B^{\prime}}=\overrightarrow{O A^{\prime}}+\left[\frac{\left(\overrightarrow{O B^{*}}-\overrightarrow{O A^{\prime}}\right) \cdot \hat{n}_{B^{*}}}{\hat{b}_{1} \cdot \hat{n}_{B^{*}}}\right] \hat{b}_{1}$

The position of incidence point $C^{\prime}$ is obtained in a similar fashion:

$\overrightarrow{O C^{\prime}}=\overrightarrow{O B^{\prime}}+\left|\overrightarrow{B^{\prime} C^{\prime}}\right| \hat{b}_{2}$

$\overrightarrow{O C^{\prime}}=\overrightarrow{O C^{*}}+\overrightarrow{C^{*} C^{\prime}}$

$\overrightarrow{C^{*} C^{\prime}} \cdot \hat{n}_{C^{*}}=0$

leading to:

$\overrightarrow{O C^{\prime}}=\overrightarrow{O B^{\prime}}+\left[\frac{\left(\overrightarrow{O C^{*}}-\overrightarrow{O B^{\prime}}\right) \cdot \hat{n}_{C^{*}}}{\hat{b}_{2} \cdot \hat{n}_{C^{*}}}\right] \hat{b}_{2}$

The points $B^{\prime}$ and $C^{\prime}$ are also shown in Figure $2 \mathrm{~b}$. The point $T^{\prime}$ in the target plane is found in slightly simpler fashion from:

$\overrightarrow{O T^{\prime}}=\overrightarrow{O C^{\prime}}+\left|\overrightarrow{C^{\prime} T^{\prime}}\right| \hat{b}_{3}$

$\overrightarrow{O T^{\prime}} \cdot \hat{z}=0$

leading to: 
$\overrightarrow{O T^{\prime}}=\overrightarrow{O C^{\prime}}-\left[\frac{\overrightarrow{O C^{\prime}} \cdot \hat{z}}{\hat{b}_{3} \cdot \hat{z}}\right] \hat{b}_{3}$

Total measured velocity is the sum of the measured velocities from points $B^{\prime}, C^{\prime}$ and $T^{\prime}$ and, from equations $(2 \mathrm{a} \& \mathrm{~b})$, is written as:

$U_{m}=\left(\hat{b}_{2}-\hat{b}_{1}\right) \cdot \overrightarrow{V_{B^{\prime}}}+\left(\hat{b}_{3}-\hat{b}_{2}\right) \cdot \overrightarrow{V_{C^{\prime}}}-\hat{b}_{3} \cdot \overrightarrow{V_{T^{\prime}}}$

The measured target velocity is given by equation (4b) and, based on equation (5), the surface velocities for the optical devices can be written as:

$\overrightarrow{V_{B^{\prime}}}=\dot{\gamma}_{1} \hat{z}_{1} \times \overrightarrow{B^{*} B^{\prime}}$

$\overrightarrow{V_{C^{\prime}}}=\dot{\alpha}_{2} \hat{x}_{2} \times \overrightarrow{C^{*} C^{\prime}}$

As presented to this point, the model is totally general. The particular path scanned depends on the functions used for mirror scan angles $\gamma_{1}$ and $\alpha_{2}$. Constant values result in the traditional point-bypoint scanning measurements while line scans and area scans are enabled by appropriate time-varying functions. If the intention is to track a point on a target rotating at angular speed $\Omega_{T}$ around the z-axis then suitable functions are:

$\gamma_{1}=\frac{\pi}{4}+0.5\left(\tan ^{-1} \frac{r_{S}}{d_{S}+z_{A}}\right) \sin \left(\Omega_{T} t+\phi_{m}\right)$

$\alpha_{2}=\frac{\pi}{4}+0.5\left(\tan ^{-1} \frac{r_{S}}{z_{A}}\right) \cos \left(\Omega_{T} t+\phi_{m}\right)$

where $t$ is time and $\phi_{m}$ is an initial phase determining the initial mirror orientations and thereby allowing any start point in the scan. The different amplitudes compensate for the separation between the mirrors, resulting in improved circularity in the scan path. It is a straightforward matter to add angular errors, for example in mean orientation or in the amplitudes of oscillation, into these expressions.

\section{Dove prism SLDV}

The Dove prism is well-known as an image derotator in optical systems. This SLDV has been designed specifically for tracking measurements on a rotating component to which it brings the particular advantage that it need rotate at only half of the target rotation frequency. Without misalignments, it is possible to define the points $A$ and $B$ which correspond respectively to the 
nominal position of the laser source and the point on the rotation axis of the prism on the first face. Important points at the mid-point of the long base of the prism, $C^{*}$ (with misalignment), and along the rotation axis of the prism on the second face, $D^{*}$ (with misalignment), will be defined shortly in terms of the point $B^{*}$. With the geometry shown in Figure 3a, points $A$ and $B$ are written as:

$\overrightarrow{O A}=\left[\begin{array}{lll}\hat{x} & \hat{y} & \hat{z}\end{array}\right]\left[\begin{array}{lll}0 & 0 & z_{A}\end{array}\right]^{T}$

$\overrightarrow{O B}=\left[\begin{array}{lll}\hat{x} & \hat{y} & \hat{z}\end{array}\right]\left[\begin{array}{lll}0 & 0 & z_{B}\end{array}\right]^{T}$

With translational misalignments, the modified vectors $\overrightarrow{O A^{\prime}}$ and $\overrightarrow{O B^{*}}$ are written in the same way as equations (13a\&b) in which it is reasonable to set $\Delta z_{A}=0$. These points are shown in Figure $3 \mathrm{~b}$. The beam path is affected by refraction at the first face of the prism, reflection at the long base of the prism and a second refraction at the second face of the prism. The surface normals are written in terms of initial alignments, based on the prism orientation in Figure 3a, modified for the slope of each face and then by a rotation matrix for the prism rotation $\gamma_{1}$ around its BD axis. The normals are then further modified by angular misalignments $\beta_{1}$ and $\alpha_{1}$ around $y$ - and then $x$-directions to give:

$$
\begin{aligned}
& \hat{n}_{B^{*}}=\left[\begin{array}{lll}
\hat{x} & \hat{y} & \hat{z}
\end{array}\right]\left[x, \alpha_{1}\right]\left[y, \beta_{1}\right]\left[z, \gamma_{1}\right]\left[x,-\frac{\pi}{4}\right]\left[\begin{array}{lll}
0 & 0 & 1
\end{array}\right]^{T} \\
& \hat{n}_{C^{*}}=\left[\begin{array}{lll}
\hat{x} & \hat{y} & \hat{z}
\end{array}\right]\left[x, \alpha_{1}\right]\left[y, \beta_{1}\right]\left[z, \gamma_{1}\right]\left[\begin{array}{lll}
0 & 1 & 0
\end{array}\right]^{T} \\
& \hat{n}_{D^{*}}=\left[\begin{array}{lll}
\hat{x} & \hat{y} & \hat{z}
\end{array}\right]\left[x, \alpha_{1}\right]\left[y, \beta_{1}\right]\left[z, \gamma_{1}\right]\left[x, \frac{\pi}{4}\right]\left[\begin{array}{lll}
0 & 0 & 1
\end{array}\right]^{T}
\end{aligned}
$$

In similar fashion, the misalignments of the prism cause its rotation axis unit vector to deviate from $\hat{z}$ as follows:

$\hat{z}_{1}=\left[\begin{array}{lll}\hat{x} & \hat{y} & \hat{z}\end{array}\right]\left[x, \alpha_{1}\right]\left[y, \beta_{1}\right]\left[\begin{array}{lll}0 & 0 & 1\end{array}\right]^{T}$

from which the positions of points $C^{*}$ and $D^{*}$, also shown in Figure $3 \mathrm{~b}$, follow:

$$
\begin{aligned}
& \overrightarrow{O C^{*}}=\overrightarrow{O B^{*}}-0.5|\overrightarrow{B D}| \hat{z}_{1}-h \hat{n}_{C^{*}} \\
& \overrightarrow{O D^{*}}=\overrightarrow{O B^{*}}-|\overrightarrow{B D}| \hat{z}_{1}
\end{aligned}
$$

where $h$ is the prism half-height. With an initial laser beam orientation in the negative $z$-direction modified by angular misalignments $\beta_{L}$ and $\alpha_{L}$ around $y$ - and then $x$-directions, the expression for the initial beam orientation, $\hat{b}_{1}$, is found: 
$\hat{b}_{1}=\left[\begin{array}{lll}\hat{x} & \hat{y} & \hat{z}\end{array}\right]\left[x, \alpha_{L}\right]\left[y, \beta_{L}\right]\left[\begin{array}{lll}0 & 0 & -1\end{array}\right]^{T}$

Equations $(6 \mathrm{a} \& \mathrm{~b})$ then provide the beam orientations, $\hat{b}_{2}$ to $\hat{b}_{4}$, after each refraction or reflection as follows:

$\hat{b}_{2}=\left(\hat{b}_{1}-\left(\hat{b}_{1} \cdot \hat{n}_{B^{*}}\right) \hat{n}_{B^{*}}\right) \frac{\varepsilon_{0}}{\varepsilon_{1}}-\left(\sqrt{1-\left(\frac{\varepsilon_{0}}{\varepsilon_{1}}\right)^{2}\left(1-\left(\hat{b}_{1} \cdot \hat{n}_{B^{*}}\right)^{2}\right)}\right) \hat{n}_{B^{*}}$

$\hat{b}_{3}=\hat{b}_{2}-2\left(\hat{b}_{2} \cdot \hat{n}_{C^{*}}\right) \hat{n}_{C^{*}}$

$\hat{b}_{4}=\left(\hat{b}_{3}-\left(\hat{b}_{3} \cdot \hat{n}_{D^{*}}\right) \hat{n}_{D^{*}}\right) \frac{\varepsilon_{1}}{\varepsilon_{0}}-\left(\sqrt{1-\left(\frac{\varepsilon_{1}}{\varepsilon_{0}}\right)^{2}\left(1-\left(\hat{b}_{3} \cdot \hat{n}_{D^{*}}\right)^{2}\right)}\right) \hat{n}_{D^{*}}$

in which $\varepsilon_{0}$ and $\varepsilon_{1}$ are the refractive indeces for air and the wedge respectively. The positions of incidence points $B^{\prime}$ and $C^{\prime}$ are obtained and written in precisely the same way as equations (21d) and (22d) while incidence point $D^{\prime}$ follows in the same fashion:

$\overrightarrow{O D^{\prime}}=\overrightarrow{O C^{\prime}}+\left|\overrightarrow{C^{\prime} D^{\prime}}\right| \hat{b}_{3}$

$\overrightarrow{O D^{\prime}}=\overrightarrow{O D^{*}}+\overrightarrow{D^{*} D^{\prime}}$

$\overrightarrow{D^{*} D^{\prime}} \cdot \hat{n}_{D^{*}}=0$

leading to:

$\overrightarrow{O D^{\prime}}=\overrightarrow{O C^{\prime}}+\left[\frac{\left(\overrightarrow{O D^{*}}-\overrightarrow{O C^{\prime}}\right) \cdot \hat{n}_{D^{*}}}{\hat{b}_{3} \cdot \hat{n}_{D^{*}}}\right] \hat{b}_{3}$

The points $B^{\prime}, C^{\prime}$ and $D^{\prime}$ are shown in Figure $3 \mathrm{~b}$. The point $T^{\prime}$ in the target plane is found in the same fashion as in equations (23a-c) but is written in terms of vectors $\overrightarrow{O D^{\prime}}$ and $\hat{b}_{4}$ :

$\overrightarrow{O T^{\prime}}=\overrightarrow{O D^{\prime}}-\left[\overrightarrow{O D^{\prime} \cdot \hat{z}}\right] \hat{b}_{4}$

Total measured velocity is the sum of the measured velocities from points $B^{\prime}, C^{\prime}, D^{\prime}$ and $T^{\prime}$ and is written as:

$U_{m}=\left(\hat{b}_{2}-\hat{b}_{1}\right) \cdot \overrightarrow{V_{B^{\prime}}}+\left(\hat{b}_{3}-\hat{b}_{2}\right) \cdot \overrightarrow{V_{C^{\prime}}}+\left(\hat{b}_{4}-\hat{b}_{3}\right) \cdot \overrightarrow{V_{D^{\prime}}}-\hat{b}_{4} \cdot \overrightarrow{V_{T^{\prime}}}$ 
The measured target velocity is given by equation (4b) and the surface velocities at each deflection point are given by:

$\overrightarrow{V_{B^{\prime}}}=\dot{\gamma}_{1} \hat{z}_{1} \times \overrightarrow{B^{*} B^{\prime}}$

$\overrightarrow{V_{C^{\prime}}}=\dot{\gamma}_{1} \hat{z}_{1} \times \overrightarrow{B^{*} C^{\prime}}$

$\overrightarrow{V_{D^{\prime}}}=\dot{\gamma}_{1} \hat{z}_{1} \times \overrightarrow{D^{*} D^{\prime}}$

As presented to this point, the model is totally general. Use in the rotor tracking application requires:

$\gamma_{1}=0.5 \Omega_{T} t+\phi_{D p}$

in which $\phi_{D p}$ is an initial phase allowing for any initial angular position of the prism. An initial alignment resulting in a scan of appropriate dimensions is achieved either by translational offset(s), $x_{A}$ and/or $y_{A}$, of the laser source modifying point A:

$\overrightarrow{O A}=\left[\begin{array}{lll}\hat{x} & \hat{y} & \hat{z}\end{array}\right]\left[\begin{array}{lll}x_{A} & y_{A} & z_{A}\end{array}\right]^{T}$

to create a scan of radius $\sqrt{x_{A}^{2}+y_{A}^{2}}$ or by an angular offset of the laser source, in which at least one of the initial orientations $\alpha_{L 0}$ and $\beta_{L 0}$ is non-zero, which can be written:

$\hat{b}_{1}=\left[\begin{array}{lll}\hat{x} & \hat{y} & \hat{z}\end{array}\right]\left[x, \alpha_{L}\right]\left[y, \beta_{L}\right]\left[x, \alpha_{L 0}\right]\left[y, \beta_{L 0}\right]\left[\begin{array}{lll}0 & 0 & -1\end{array}\right]^{T}$

to produce a scan diameter dependent on the prism dimensions and $z_{B}$.

For the dual mirror and Dove prism SLDV systems, there may be situations where it is preferable to consider alignment between an optical head and the target rather than the alignment of each individual component within the optical head relative to the target. This case is considered in Appendix B.

\section{Self tracking LDV}

The self-tracking system modelled here incorporates a vertex mirror fixed to the rotor, reflecting the outgoing beam through $\pi / 2$ rad onto a conical mirror which is fixed and located directly in front of the target. A cone angle of $\theta_{c}=\pi / 4 \mathrm{rad}$ results in a second $\pi / 2 \mathrm{rad}$ reflection at the conical mirror, directing the beam onto the target surface in a direction parallel with the target rotation axis, as shown in figure 4a. In a system without misalignments, it is possible to define the points $A, B$ and $D$ which 
correspond respectively to the nominal position of the laser source, the intersection between the vertex mirror surface and the target rotation axis, and the peak of the cone of which the conical mirror is a section. Points $A$ and $B$ are described in the same way as in equations (27a\&b) while point $D$ is written in the same fashion:

$\overrightarrow{O D}=\left[\begin{array}{lll}\hat{x} & \hat{y} & \hat{z}\end{array}\right]\left[\begin{array}{lll}0 & 0 & z_{D}\end{array}\right]^{T}$

Position vector $\overrightarrow{O D}$ controls the scan radius, $\mathrm{r}_{\mathrm{S}}$, as follows:

$\mathrm{r}_{\mathrm{S}}=\tan \theta_{c}\left(\mathrm{z}_{\mathrm{D}}-\mathrm{z}_{\mathrm{B}}\right)$

With translational misalignments and noting that the $z$-position of point $A$ is nominal, the positions of points $A, B$ and $D$ are modified as follows:

$\overrightarrow{O A^{\prime}}=\overrightarrow{O A}+\left[\begin{array}{lll}\hat{x} & \hat{y} & \hat{z}\end{array}\right]\left[\begin{array}{lll}\Delta x_{A} & \Delta y_{A} & 0\end{array}\right]^{T}$

$\overrightarrow{O B^{*}}=\overrightarrow{O O^{*}}+\left[\begin{array}{lll}\hat{x} & \hat{y} & \hat{z}\end{array}\right]\left[x, \alpha_{1}+\theta_{x}\right]\left[y, \beta_{1}+\theta_{y}\right]\left[\begin{array}{lll}0 & 0 & z_{B}+\Delta z_{B}\end{array}\right]^{T}$

$\overrightarrow{O D^{*}}=\overrightarrow{O D}+\left[\begin{array}{lll}\hat{x} & \hat{y} & \hat{z}\end{array}\right]\left[\begin{array}{lll}\Delta x_{D} & \Delta y_{D} & \Delta z_{D}\end{array}\right]^{T}$

The position of point $B$ has been modified not only by the translational misalignment $\Delta z_{B}$ but also by angular misalignments $\alpha_{1}$ and $\beta_{1}$ in the mounting of the mirror and by the vibrations of the element of the target to which the mirror is fixed: translational displacement $\overrightarrow{O O^{*}}$, angular displacement $\theta_{x}$ around the $x$-axis, and angular displacement $\theta_{y}$ around the $y$-axis. This influence of vibration is a significant difference compared to the previous two systems and is the direct result of attachment of the vertex mirror to the structure of interest. These misaligned reference points are shown in Figure $4 \mathrm{~b}$. The surface normal for the vertex mirror, $\hat{n}_{B^{*}}$, is written in terms of an initial alignment in the positive $z$-direction modified by successive rotation matrices for surface inclination $-\pi / 4$, rotation $\gamma_{1}$, and the same combination of angular misalignments and angular vibrations as in equation (42b):

$\hat{n}_{B^{*}}=\left[\begin{array}{lll}\hat{x} & \hat{y} & \hat{z}\end{array}\right]\left[x, \alpha_{1}+\theta_{x}\right]\left[y, \beta_{1}+\theta_{y}\right]\left[z, \gamma_{1}\right]\left[x,-\frac{\pi}{4}\right]\left[\begin{array}{lll}0 & 0 & 1\end{array}\right]^{T}$

$\gamma_{1}$ is written in terms of an initial angular position, $\gamma_{01}$ :

$\gamma_{1}=\Omega_{\mathrm{T}} \mathrm{t}+\gamma_{01}$ 
The initial laser beam orientation is written in the same way as equation (31) and orientation after the vertex mirror reflection is written in the same way as equation (19), while that after the conical mirror reflection is written:

$\hat{b}_{3}=\hat{b}_{2}-2\left(\hat{b}_{2} \cdot \hat{n}_{C^{\prime}}\right) \hat{n}_{C^{\prime}}$

Equation (44) highlights another significant difference between this analysis and those for the previous systems. Use of the conical mirror rather than a plane mirror means that surface normal is a function of the specific point of incidence on the cone and equation (9) does not hold. This apparently simple distinction has major implications. In the previous analyses, the surface normal itself would be used to identify the point of incidence but, for this self-tracking system, the point of incidence must be determined first in order to define the surface normal and so additional steps are required. To proceed, cone axes with associated unit vectors $\hat{x}_{c o n}, \hat{y}_{c o n}$ and $\hat{z}_{c o n}$ are defined. These directions are the result of angular misalignment of the cone axes by $\beta_{2}$ and then $\alpha_{2}$ around $y$-and then $x$-directions. A coordinate transformation can be made according to:

$\left[\begin{array}{lll}\hat{x}_{\text {con }} & \hat{y}_{\text {con }} & \hat{z}_{\text {con }}\end{array}\right]=\left[\begin{array}{lll}\hat{x} & \hat{y} & \hat{z}\end{array}\right]\left[x, \alpha_{2}\right]\left[y, \beta_{2}\right]\left[\begin{array}{lll}1 & 0 & 0 \\ 0 & 1 & 0 \\ 0 & 0 & 1\end{array}\right]$

The orientation of the surface normal vector $\hat{n}_{C^{\prime}}$ is illustrated in figure $5 \mathrm{a}$ and can be described by an initial orientation in the $-z_{c o n}$-direction followed by a negative rotation by the cone angle $\pi / 4$ rads around $x_{c o n}$, followed by a rotation around $z_{c o n}$ by the currently unknown angle $\gamma_{c o n}$ :

$\hat{n}_{C^{\prime}}=\left[\begin{array}{lll}\hat{x}_{\text {con }} & \hat{y}_{\text {con }} & \hat{z}_{\text {con }}\end{array}\right]\left[z_{\text {con }}, \gamma_{\text {con }}\right]\left[x_{\text {con }},-\frac{\pi}{4}\right]\left[\begin{array}{lll}0 & 0 & -1\end{array}\right]^{T}$

Incorporating the coordinate transformation of equation (45) gives:

$\hat{n}_{C^{\prime}}=\left[\begin{array}{lll}\hat{x} & \hat{y} & \hat{z}\end{array}\right]\left[x, \alpha_{2}\right]\left[y, \beta_{2}\right]\left[\hat{z}_{c o n}, \gamma_{c o n}\right]\left[\hat{x}_{c o n},-\frac{\pi}{4}\right]\left[\begin{array}{lll}0 & 0 & -1\end{array}\right]^{T}$

While the position of incidence point $B^{\prime}$ is obtained and written in the same way as in equation (21d), the position of incidence point $C^{\prime}$ cannot be obtained from the usual three vector expressions:

$$
\begin{aligned}
& \overrightarrow{O C^{\prime}}=\overrightarrow{O B^{\prime}}+\left|\overrightarrow{B^{\prime} C^{\prime}}\right| \hat{b}_{2} \\
& \overrightarrow{O C^{\prime}}=\overrightarrow{O D^{*}}+\overrightarrow{D^{*} C^{\prime}} \\
& \overrightarrow{D^{*} C^{\prime}} \cdot \hat{n}_{C^{\prime}}=0
\end{aligned}
$$


because in equation (47c) both vectors are unknown. A first equation for $\overrightarrow{D^{*} C^{\prime}}$ can be written by combination of equations $(47 \mathrm{a} \& \mathrm{~b})$ :

$\overrightarrow{D^{*} C^{\prime}}=\overrightarrow{O B^{\prime}}-\overrightarrow{O D^{*}}+\left|\overrightarrow{B^{\prime} C^{\prime}}\right| \hat{b}_{2}$

A second is provided by considering $\overrightarrow{D^{*} C^{\prime}}$ within its cone. The orientation of $\overrightarrow{D^{*} C^{\prime}}$ is illustrated in figure $5 \mathrm{~b}$ and can be described by an initial orientation in the $-\hat{z}_{c o n}$-direction followed by a rotation by the cone angle, $\pi / 4$ rads, around $\hat{x}_{c o n}$, followed by a rotation around $\hat{z}_{c o n}$ by $\gamma_{c o n}$ :

$\frac{\overrightarrow{D^{*} C \prime}}{\left|\overrightarrow{D^{*} C \mid}\right|}=\left[\begin{array}{lll}\hat{x}_{c o n} & \hat{y}_{\text {con }} & \hat{z}_{c o n}\end{array}\right]\left[\hat{z}_{c o n}, \gamma_{c o n}\right]\left[\hat{x}_{c o n}, \frac{\pi}{4}\right]\left[\begin{array}{lll}0 & 0 & -1\end{array}\right]^{T}$

On expansion, this provides:

$\overrightarrow{D^{*} C^{\prime}}=\frac{\left|\overrightarrow{D^{*} C \prime}\right|}{\sqrt{2}}\left[\begin{array}{lllll}\hat{x}_{c o n} & \hat{y}_{\text {con }} & \hat{z}_{\text {con }}\end{array}\right]\left[\begin{array}{lll}-\sin \gamma_{\text {con }} & \cos \gamma_{\text {con }} & -1\end{array}\right]^{T}$

Substituting equation (45) into equation (49b), expanding and making small angle approximations for misalignment angles (including neglecting terms with products of misalignment angles) results in:

$\overrightarrow{D^{*} C^{\prime}}=\frac{\left|\overrightarrow{D^{*} C^{\prime}}\right|}{\sqrt{2}}\left[\begin{array}{lll}\hat{x} & \hat{y} & \hat{z}\end{array}\right]\left[-\left(\beta_{2}+\sin \gamma_{c o n}\right) \quad\left(\alpha_{2}+\cos \gamma_{c o n}\right) \quad\left(\beta_{2} \sin \gamma_{c o n}+\alpha_{2} \cos \gamma_{c o n}-1\right)\right]^{T}(49 \mathrm{c})$

On combining equations (48) and (49c), the resulting expression has 3 unknowns, $\left|\overrightarrow{D^{*} C^{\prime}}\right|, \gamma_{c o n}$ and $\left|\overrightarrow{B^{\prime} C^{\prime}}\right|$ which can be found by equating the three components of the vector equation. Finding $\gamma_{c o n}$ enables expression of the surface normal at $C^{\prime}$ according to equation (46b). Finding $\left|\overrightarrow{B^{\prime} C^{\prime}}\right|$ identifies the final deflection point $C^{\prime}$ from equation (47a). Algebraic manipulation to find $\gamma_{c o n}$ and $\left|\overrightarrow{B^{\prime} C^{\prime}}\right|$ is presented in Appendix C.

Attention must now be paid to the way that the laser beam scans repeatedly around the conical mirror. Equation (46b) effectively assumes that the conical mirror has perfectly circular cross-sections. It is, however, likely that there will be some variation from the circular profile that will introduce a pseudorandom noise into the expression for surface normal. This can be written in terms of continuously varying angular misalignments, $\alpha_{c m}$ and $\beta_{c m}$, around the $x_{c m}$ and $y_{c m}$ axes respectively. As shown in figure $5 \mathrm{c}$, these axes rotate with the beam around the conical mirror such that the $z_{c m}$ axis is always aligned with the surface normal (without these misalignments) but opposite to it. These axes can then be transformed into the cone axes using the rotation matrices $\left[z_{\text {con }}, \gamma_{\text {con }}\right]$ and $\left[x_{\text {con }},-\frac{\pi}{4}\right]$ and the result then transformed into the global axes using equation (45) as follows: 
$\hat{n}_{C^{\prime}}=\left[\begin{array}{lll}\hat{x}_{c m} & \hat{y}_{c m} & \hat{z}_{c m}\end{array}\right]\left[x_{c m}, \alpha_{c m}\right]\left[y_{c m}, \beta_{c m}\right]\left[\begin{array}{lll}0 & 0 & -1\end{array}\right]^{T}$

$\hat{n}_{C^{\prime}}=\left[\begin{array}{lll}\hat{x} & \hat{y} & \hat{z}\end{array}\right]\left[x, \alpha_{2}\right]\left[y, \beta_{2}\right]\left[z_{c o n}, \gamma_{c o n}\right]\left[x_{c o n},-\frac{\pi}{4}\right]\left[x_{c m}, \alpha_{c m}\right]\left[y_{c m}, \beta_{c m}\right]\left[\begin{array}{lll}0 & 0 & -1\end{array}\right]^{T}$

As the beam takes the same path around the mirror with each rotation, these surface profile variations are periodic and have the form:

$\alpha_{c m}=\sum_{n=1}^{N} A_{\alpha n} \sin \left(n \Omega_{T}+\phi_{\alpha n}\right)$

$\beta_{c m}=\sum_{n=1}^{N} A_{\beta n} \sin \left(n \Omega_{T}+\phi_{\beta n}\right)$

in which $N \Omega_{T}$ is capped at the Nyquist frequency in any frequency analysis undertaken. With $\hat{n}_{C^{\prime}}$ now expressed fully, the final beam orientation is found from equation (44). The solution is completed by using $\overrightarrow{O C^{\prime}}$ and $\hat{b}_{3}$ to find the intersection of the beam in the $x y$ plane as in equation (23c).

As the conical mirror is stationary, the total measured velocity is the sum of the measured velocities from points $B^{\prime}$ and $T^{\prime}$ and, from equations ( $\left.2 \mathrm{a} \& \mathrm{~b}\right)$, is written as:

$U_{m}=\left(\hat{b}_{2}-\hat{b}_{1}\right) \cdot \overrightarrow{V_{B^{\prime}}}-\hat{b}_{3} \cdot \overrightarrow{V_{T^{\prime}}}$

The measured target velocity is given by equation (4b). For the vertex mirror, target vibrations must also be included in its surface velocity which, for the set of motions under consideration and based on equation (5), can be written as:

$\overrightarrow{V_{B^{\prime}}}=V_{O^{*} x} \hat{x}+\dot{\gamma}_{1} \hat{z} \times \overrightarrow{B^{*} B^{\prime}}$

\section{Implementation of the Models}

In assessing these systems, the main areas of interest are deviations between the desired and actual scan paths and three distinct features of the total measured velocity. These features are the effects of misalignments of the optical system in the presence of target rotation, the sensitivity to target out-ofplane vibration (the intended measurement), and the sensitivity to target in-plane vibration (an 
undesired additional measured velocity). The models are perfectly suited to such an in-depth evaluation. With each system configured to scan a circle with radius $50 \mathrm{~mm}$ centred on the fixed origin $O$, this is exemplified by the following close scrutiny of the quality of the scanned circles. The dual mirror SLDV with corrected scan amplitudes is well centred with only very small deviation from the circular path - a standard deviation on radial position of a mere $0.002 \%$ of scan radius. The Dove prism SLDV, with an initial angular alignment of the laser beam, delivers a scan with a small offset from the optical axis ( $0.6 \%$ of scan radius) with a standard deviation on radial position of $0.06 \%$ of scan radius. The self-tracking system scans a perfectly centred, perfect circle.

In the simulations, vibration frequencies are specifically chosen to allow the effects of misalignment, out-of-plane vibration and in-plane vibration to be distinguished from one another. Of course, there is no such luxury in an actual measurement and the challenge in data interpretation will be compounded. Particular attention is paid to whether the Doppler shifts (shown as velocities) contributing to the total measured velocity originate at optical devices or at the target and to their combination which can be constructive or destructive. This emphasises the comprehensive detail available through these models. Figures 6-8 show indicative spectral content (presented against target rotation order) of the individual contributions to the total measured velocity (normalised by target rotation speed) for single misaligned configurations. Velocity spectra in the absence of misalignment are not shown but they are discussed and data are included in Tables 1-3. These tables summarise simulations of a very large number of misaligned configurations for each system. Realistic misalignment values are used at all times (as listed in Appendix D) but the resulting scans are also checked to ensure that scan radius (defined as the mean radial position from the scan centre location) is within $5 \%$ of the intended 50 $\mathrm{mm}$ and that the scan centre location itself is less than $5 \%$ of the scan radius from its intended location. Only scans passing these tests are used for the analysis summarised in Tables 1-3. The second column in each of these tables indicates how many scans were used from the total number of scans considered. These thresholds could be debated but they are indicative of what might reasonably be expected in an actual measurement and, most importantly, they ensure fairness in the comparison. Table 1 presents spectral content associated with system configuration (i.e. in the absence of misalignments) and with misalignments. These velocities depend on target rotation but not target vibration. Tables 2 and 3 show, respectively, the sensitivities to flexible out-of-plane vibration and whole body in-plane vibration. Sensitivities are seen not only at the vibration frequencies but also at sidebands related to rotation speed. In the case of out-of-plane vibration, sidebands are symmetrical but this is not the case for in-plane vibration where sideband amplitudes are in the ratio of their frequencies. The origin of this effect is in the combination of scanning and target rotation and is readily demonstrated by evaluation of equation (4c) for any of the models, as set out in Appendix E. The order and frequency ranges used in the Tables are chosen to cover all measurable spectral peaks. 
The simulations do not include typical measurement noise but, as important context, noise , especially that due to laser speckle, would make identification of an individual spectral peak difficult if its amplitude were less than $0.2 \mu \mathrm{m} / \mathrm{s} / \mathrm{rad} / \mathrm{s}$ [16]. Vibration amplitudes of $1 \mathrm{~mm} / \mathrm{s} / \mathrm{rad} / \mathrm{s}$ are used in the simulations; on the same basis sensitivities greater than $0.02 \%$ should be visible in measured spectra but this value is obviously dependent on vibration amplitude. This noise level has influenced the scales chosen for figures 6-8 but all peak amplitudes, of any level, are given in Tables 1-3 where emboldened figures indicate spectral peaks that ought to be observable in real measurement data.

\subsection{Dual Mirror SLDV simulation}

Without misalignments, no Doppler shifts occur at the mirror surfaces but there is a prominent Doppler shift from the target at second order (and much smaller fourth and sixth order components). There is $99.9 \%$ measurement sensitivity to out-of-plane vibrations, with very small sidebands apparent at $\omega_{\mathrm{z}} \pm 2 \Omega_{\mathrm{T}}$, while the sensitivity to in-plane vibration is principally characterised by peaks of unequal amplitude at frequencies $\omega_{x} \pm \Omega_{T}$. By average amplitude, these peaks correspond to $2 \%$ in-plane sensitivity.

For the specific misalignments listed, Figure 6a shows velocities associated with the deflections at each mirror surface while Figure $6 \mathrm{~b}$ shows the combination of velocities from mirrors and target to produce the total measured velocity. The small deviations from the intended circular scan path would not be visible in a figure but, with these misalignments, the scan path has a mean radial position of $99.9 \%$ (standard deviation $0.2 \%$ ) and a centre position error of $1.5 \%$, relative to the intended $50 \mathrm{~mm}$ radius. Figure 6a shows how misalignments cause Doppler shifts at both mirrors, principally at first order with much smaller DC, $2 \mathrm{x}$ and $3 \mathrm{x}$ components generated at the second mirror across whose surface the beam scans. Additional Doppler shifts also occur at the target, principally at DC and first order with a much smaller $3 \mathrm{x}$ component. Figure $6 \mathrm{~b}$ also shows the influence of misalignments on the measured velocity components associated with target vibration. Additional sidebands appear at $\pm \Omega_{\mathrm{T}}$ around the $z$-vibration frequency, $\omega_{\mathrm{z}}=8 \Omega_{\mathrm{T}}$, while an additional spectral peak occurs at the in-plane vibration frequency, $\omega_{x}=16 \Omega_{\mathrm{T}}$.

Based on 50621 misaligned configurations, Table 1 shows that peaks at first and second order are the most significant amongst the components unrelated to vibrations. Standard deviations indicate that the first order component varies considerably with misalignment unlike the second order component which is largely unaffected. The principal sensitivity to out-of-plane vibration at $\omega_{z}$ and the sidebands at $\omega_{x} \pm \Omega_{T}$ associated with in-plane vibrations are unaffected by misalignment, as shown in Tables 2 and 3 respectively. Misalignments do, however, generate a peak at the in-plane vibration frequency 
with a measurable mean level and a standard deviation that indicates substantial dependence on those misalignments.

The effect of changing scan radius has also been explored using the same set of misaligned configurations. Doubling scan radius by doubling scan angles leaves the DC level unchanged but doubles the $1 \mathrm{x}$ velocity and quadruples the $2 \mathrm{x}$ velocity. Vibration sensitivities at sidebands double but out-of-plane vibration sensitivity at $\omega_{z}$ reduces only negligibly and in-plane vibration sensitivity at $\omega_{x}$ is unchanged. Doubling scan radius by doubling the axial distance $z_{A}$ leaves the DC level and the sensitivities at $\omega_{z}$ and $\omega_{x}$ unchanged. Levels increase at first order, second order, $\omega_{z} \pm \Omega_{T}$ and $\omega_{x} \pm \Omega_{T}$ only modestly, representing negligible differences in sensitivity and indicating the desirability of managing scan radius by maximising $z_{A}$ and minimising scan angles.

\subsection{Dove prism SLDV simulation}

Scanning in a circular path can be arranged by initial translational or angular alignment of the laser beam. For translational alignment (no misalignments), the $1 \mathrm{x}$ velocity components associated with each of the three deflections at the prism cancel completely on addition and no measured velocity originates at the target at low orders. There is $100 \%$ sensitivity to out-of-plane vibrations and no sensitivity at all to in-plane vibrations. However, the finite dimension of the prism means that only a small scan radius can be achieved in this way and an initial angular alignment of the laser beam is the more practical option. With angular alignment (no misalignments), spectral peaks at integer multiples of half order up to order 3.5 appear for each deflection at the Dove prism and from the target. Halforder multiples appear because the Dove prism rotates at half of the target rotation speed. From the prism deflections, peaks at $0.5 \mathrm{x}$ and $1 \mathrm{x}$ components have the largest amplitudes but there is significant cancellation, especially at half order, on addition. The largest combined peak is at first order but it is small at around $4 \mu \mathrm{m} / \mathrm{s} / \mathrm{rad} / \mathrm{s}$. There is $99.9 \%$ measurement sensitivity to out-of-plane vibrations, with no sidebands, while the sensitivity to in-plane vibration is again characterised by peaks of unequal amplitude at frequencies $\omega_{x} \pm \Omega_{T}$. By average amplitude, these peaks correspond to slightly less than $2 \%$ in-plane sensitivity.

For the specific misalignments listed, Figure 7a shows velocities associated with the deflections at each prism deflection point while Figure $7 \mathrm{~b}$ shows the combination of velocities on the prism, velocity at the target and the total measured velocity. With these misalignments, the scan path has a mean radial position of $96.4 \%$ (standard deviation $0.06 \%$ ) and a centre position error of $2.3 \%$, relative to the intended $50 \mathrm{~mm}$ radius. The circular scans from consecutive prism half-rotations follow paths that are separated by around $0.1 \%$ of radius. This is driven by the initial angular alignment and is largely unaffected by misalignments. Its practical implications are negligible. Figure 7 a shows the 
addition of DC peaks and considerably larger 1x components for each of the prism deflection points but, again, there is significant cancellation on addition. Figure $7 \mathrm{~b}$ shows how the dominant DC peak from the prism overall cancels significantly with that from the target, leaving the $1 \mathrm{x}$ component generated at the target as the dominant low order component in the total measured velocity. The sensitivities to out-of-plane and in-plane vibrations shown in Figure $7 \mathrm{~b}$ follow a very similar pattern to those found in the dual mirror SLDV system with the addition of sidebands at $\omega_{z} \pm \Omega_{T}$ and a peak at $\omega_{x}$.

Simulation of 15155 misaligned configurations, as summarised in Tables 1-3, reveals that the main low order components are at DC and $1 \mathrm{x}$ with mean values and standard deviations similar to those found in the dual mirror system. The peak at half order is small but measureable. The vibration sensitivities also follow similar patterns to those seen for the dual mirror system. The principal sensitivities at $\omega_{\mathrm{z}}$ and $\omega_{x} \pm \Omega_{T}$ are unaffected by misalignment, while the peak appearing at $\omega_{x}$ has measurable magnitude at $0.6 \%$, approximately twice that seen in the dual mirror system, with substantial dependence on those misalignments.

Doubling scan radius by doubling the initial alignment angle increases the mean DC level by just a few percent but increases the level at half order by a factor of 5 and almost doubles the mean level at first order. Negligible reductions are seen in the mean sensitivities at $\omega_{z}$ and $\omega_{x}$ while the sensitivities at $\omega_{\mathrm{z}} \pm \Omega_{\mathrm{T}}$ and $\omega_{x} \pm \Omega_{\mathrm{T}}$ almost double. The more desirable way to double scan radius is by doubling the axial distance $z_{B}$ which leaves all mean levels and sensitivities unchanged.

\subsection{Self-tracking LDV}

In a perfectly aligned system, there are no velocities at low orders and $100 \%$ sensitivity to out-ofplane flexible vibration. Sensitivity to whole body in-plane vibrations is again associated with peaks at $\omega_{\mathrm{x}} \pm \Omega_{\mathrm{T}}$. The amplitudes of these peaks are again in the ratio of their frequencies (see Appendix E), this time with a mean amplitude of $50 \%$. This high sensitivity occurs as a result of the whole body in-plane vibration causing Doppler shifts at the vertex mirror which is attached directly to the target. For the same reason, the sensitivity to out-of-plane whole body vibrations, $V_{O^{*}}{ }_{z} \hat{z}$, would be $200 \%$. This clearly emphasises the importance of considering Doppler shifts at optical devices. The sensitivity to in-plane vibrations is affected by the type of in-plane motion encountered and there would be zero sensitivity to in-plane vibration associated with target flexibility (i.e. $V_{f x}$ and/or $V_{f y}$ ). Together with $100 \%$ sensitivity to out-of-plane flexible vibrations, this is a major attraction of this conical mirror arrangement. For the previous two systems, sensitivities to whole body in-plane vibration and to in-plane vibration associated with target flexibility are the same because all associated Doppler shifts occur at the target. Two misalignment simulations have been performed for 
the self-tracking LDV. Simulation 1 considers misalignment of the optical components as for the previous two systems while simulation 2 additionally includes the effect of the conical mirror surface profile.

\subsubsection{Simulation 1}

Figure 8a shows the velocities associated with the deflection at the vertex mirror and the target, together with their combination to produce the total measured velocity with the misalignments listed. For this case, the scan path has a mean radial position of $102.30 \%$ (standard deviation of $1.80 \%$ ) and a centre position error of $2.74 \%$, each relative to the intended $50 \mathrm{~mm}$ scan radius. Compared to the previous two systems, misalignments appear to cause larger and more numerous spectral components including spectral peaks from DC to second order from the vertex mirror and from DC to fifth order from the target. Figure 8a illustrates a simulation with vibration frequencies of $\omega_{z}=10 \Omega_{\mathrm{T}}$ and $\omega_{x}=20 \Omega_{\mathrm{T}}$ and an expanded order range to allow individual effects to be distinguished within the larger number of spectral peaks present. (The data summarised in Tables 1-3 are still based on $\omega_{z}=8 \Omega_{\mathrm{T}}$ and $\omega_{x}=16 \Omega_{\mathrm{T}}$ ). The effect of misalignments on out-of-plane vibration sensitivity is the addition of small sidebands at up to $\omega_{\mathrm{z}} \pm 3 \Omega_{\mathrm{T}}$. With in-plane vibration, misalignments cause additional sidebands as well as the peak at the in-plane vibration frequency, itself, as seen previously.

From 180675 misaligned configurations, Table 1 shows that levels at DC and the integer orders up to $4 \mathrm{x}$ are high relative to the previous two systems with relatively large standard deviations indicating significant variation with misalignment. The components at $1 \mathrm{x}$ and $2 \mathrm{x}$ would be readily observable in measured data. The main vibration sensitivities at $\omega_{\mathrm{z}}$ and $\omega_{\mathrm{x}} \pm \Omega_{\mathrm{T}}$ are largely unaffected by misalignments with very small standard deviations, as shown in Tables 2 and 3 respectively. Table 3 shows that the largest additional vibration sensitivities appear at $\omega_{\mathrm{x}}$ and $\omega_{\mathrm{x}} \pm 2 \Omega_{\mathrm{T}}$, both the result of Doppler shifts at the target. At $2 \%$, the mean sensitivity at $\omega_{\mathrm{x}}$ is the highest of the three systems considered while the sidebands at $\omega_{\mathrm{x}} \pm 2 \Omega_{\mathrm{T}}$ have mean sensitivities similar to those seen at $\omega_{\mathrm{x}} \pm \Omega_{\mathrm{T}}$ in the previous two systems. The ratio of amplitudes for each sideband pair is comparable with the ratio of their frequencies.

Doubling the scan radius by increasing $z_{D}$ results in useful reductions in the undesired low order components with the exception of the $1 \mathrm{x}$ component which increases by $7 \%$. The DC level reduces by almost one-quarter, the $2 \mathrm{x}$ component is almost halved, and $3 \mathrm{x}$ and $4 \mathrm{x}$ components reduce by around a factor of 4 . Out-of-plane sensitivity at $\omega_{z}$ increases very slightly while its sidebands reduce by a factor of around 4 . The undesired sensitivity to in-plane vibration at $\omega_{\mathrm{x}}$ almost halves, as do the levels of the $\omega_{\mathrm{x}} \pm 2 \Omega_{\mathrm{T}}$ sidebands, but the high sidebands at $\omega_{\mathrm{x}} \pm \Omega_{\mathrm{T}}$ are largely unaffected.

\subsubsection{Simulation 2}


This conical mirror surface profile variation is not quantified by a single value like the misalignments in each individual configuration in simulation 1 but by a continuously varying pair of small rotations as shown in equation $(51 \mathrm{a} \& \mathrm{~b})$. The variation has spectral content at all orders but it is reasonable to expect higher levels at lower orders so an overall Gaussian profile has been imposed on the component amplitudes (4th order set to be $e^{-2}$ of the Gaussian). Those amplitudes also have a random component equivalent to approximately $+/-10 \%$ of their mean value and they are scaled so that the mean deviation of $\hat{n}_{C^{\prime}}$ from its unmodified orientation takes a chosen value, in this case $0.1^{\circ}$. This value would result in a deviation of the point of incidence on the target of less than $0.1 \mathrm{~mm}$ over $5 \mathrm{~cm}$. For otherwise perfect alignment and in the absence of vibration, the measured velocity would result entirely from the effect of the conical mirror surface profile on the detected component of target rotation velocity and would take the form shown in Figure $8 \mathrm{~b}$ in which the dominance of lower orders is clear.

In the additional presence of misalignments, Figure 8c shows the components of the total measured velocity. In addition to the effects discussed in the previous sub-section, the velocity associated with the target shows a pattern of additional spectral peaks that might have been expected from Figure $8 \mathrm{~b}$. At the low orders, Table 1 shows that the DC and first order levels are unaffected but, thereafter, the low orders are significantly increased. Tables 2 and 3 show that the vibration sensitivities are not fundamentally affected unless the levels associated with the surface profile are significant at the same frequencies at which vibration sensitivity is also apparent. In these data, this is the case at the sidebands associated with out-of-plane vibration because it has the lower frequency in the simulation. Those predicted values where an effect is apparent should be regarded only in order of magnitude terms. The assumptions made are all reasonable, especially the resulting mean angular deviation, but the precise values used for each order are too speculative for a more quantitative interpretation. Only one surface profile variation has been simulated; it is perfectly possible to simulate more but it is not meaningful to do this without some firmer basis to guide the selection of amplitudes for each harmonic component. For this reason, affected mean levels and sensitivities and their standard deviations are shown with only a single significant figure. Standard deviations are shown for completeness but, at low orders, they result from the combination of many individual misalignments with a dominant but single conical mirror surface profile and so they are similar to values in simulation 1 and say nothing about the effect of the conical mirror.

\subsection{Validation of the models}

An experimentally validated theoretical prediction of measured velocities at DC and the first and second orders in the presence of misalignments has been published for the dual mirror SLDV [16]. This paper presented theoretical expressions, a procedure in which initial (unknown) misalignments 
can be calculated for subsequent correction and data from real measurements. A much less comprehensive set of misalignments than have been modelled here were considered, based on angular and translational misalignment between the optical and rotor axes. The configuration is modelled by setting all misalignment variables in section 3 to zero and then incorporating misalignments in the manner set out in Appendix B. Checking against data such as those presented here in Figure 6 and the calculation of initial (unknown) misalignments in [16], there is perfect agreement between the original theory (before its use of small angle approximations) and the model, which is itself without approximation. $1 \mathrm{x}$ and $2 \mathrm{x}$ levels seen in real measurements with care taken over alignment show excellent agreement with the range indicated in Table 1, after adjustment for scan angle and axial distance. The patterns of peaks at in-plane and out-of-plane vibration frequencies and sidebands have also been seen in previous work, including the distinctive combination of a smaller peak at vibration frequency and larger sidebands of unequal amplitude at $\omega_{\mathrm{x}} \pm \Omega_{\mathrm{T}}$ associated with in-plane vibrations in the presence of misalignments [14].

The validation of the dual mirror SLDV model goes some way towards validating the models for the Dove prism and self-tracking systems because of the generic root of the individual models.

Systematic, experimental studies of the effects of misalignment in the Dove prism and self-tracking systems have not, to the authors' knowledge, been published but a comparison can still be made with available data.

For the Dove prism system, a case study on a rotating compact disc [43] shows levels in the region of $1 \mu \mathrm{m} / \mathrm{s} / \mathrm{rad} / \mathrm{s}$ at $0.5 \mathrm{x}$ and $260 \mu \mathrm{m} / \mathrm{s} / \mathrm{rad} /$ at $1 \mathrm{x}$. Geometrical details are not given but scan radius can be assumed to be in the region of 50mm given the application and these measured levels sit very comfortably with the predictions in Table 1 , noting the volatility observed in the level at $0.5 \mathrm{x}$ as discussed in section 6.2.

For the self-tracking system, after alignment, values have been reported in the region of $700 \mu \mathrm{m} / \mathrm{s}$ / $\mathrm{rad} / \mathrm{s}$ at first order and $220 \mu \mathrm{m} / \mathrm{s} / \mathrm{rad} / \mathrm{s}$ at second order for a scan radius of around $200 \mathrm{~mm}$ [44]. Table 4 shows three simulations for this scan radius in which a threshold has been set corresponding to maximum $1 \mathrm{~mm}$ error in scan centre location and scan radius. To acknowledge the alignment effort made in preparation for the experiments [44], larger individual misalignments have been eliminated from the simulation (as listed in Appendix D). The DC and 1x components are largely unaffected by the effect of the conical mirror surface profile. The measured level at first order sits at a level close to (just below) the mean minus one standard deviation in each of the simulations, consistent with the effort made in alignment. The measured level also confirms the general conclusion from the simulations that much higher first order velocities occur with this system than they do with the previous two systems. Without inclusion of the effect of conical mirror surface profile, the predicted 
second order level is an order of magnitude smaller than the measured level. Inclusion of the surface profile used in section 6.3.2 results in a second order level comparable with measured data. In the measured data, it was observed that the $3 \mathrm{x}$ component was small but the $4 \mathrm{x}$ component much higher at around $140 \mu \mathrm{m} / \mathrm{s} / \mathrm{rad} / \mathrm{s}$. A surface profile with a stronger second order variation is one possible cause of this higher level. The third simulation is based on the second simulation, with the same overall angular deviation in surface normal vector but components at 2 nd order and its integer multiples are increased by a factor of 3 . The levels predicted are now consistent with the trend in measured data, suggesting that the conical mirror surface profile may well be the cause of the effects observed in measured velocity at integer orders beyond $1 \mathrm{x}$.

\section{Conclusions}

A novel vectorial framework for modelling measured velocity in laser vibrometry has been introduced and successfully applied. The framework is comprehensive and includes the largely neglected velocity contributions associated with deflections at the optical devices in scanning heads. Universal applicability, ease of use and the ability to incorporate misalignments of every device are its key attributes and this has been demonstrated by modelling three significantly different scanning systems. The models developed have been interrogated in considerable depth, principally as a means to emphasise the framework's value but also to enable a quantitative performance comparison between systems for the rotor tracking application. Low order velocity components are the principal effect of misalignment. For the dual mirror system, measurable velocities are expected at DC, first and second order while the Dove prism system shows similar levels at DC and first order. The second order component is much smaller but there are also small peaks at half orders including a measurable component at $0.5 \mathrm{x}$. For the self-tracking system, a greater effect of misalignment is encountered at DC and integer orders, especially at $1 \mathrm{x}$ and $2 \mathrm{x}$. In particular, the surface profile of its conical mirror has the potential to increase components at integer orders above 1x. While the models can incorporate target motion of any complexity, out-of-plane flexible vibrations and in-plane whole body vibrations have been the focus of simulations. Sensitivity to out-of-plane flexible vibrations is close to $100 \%$ for all systems and largely unaffected by misalignments with the exception of the appearance of sidebands with negligible amplitude. In-plane vibration sensitivity is characterised principally by sideband peaks at $\pm \Omega_{T}$ around the vibration frequency with amplitudes in the ratio of their frequencies. Again, these are largely unaffected by misalignment but misalignment does cause a smaller peak to appear at the vibration frequency itself. For the dual mirror and the Dove prism systems, the main in-plane vibration sensitivities are small at around $2 \%$. For the self tracking system, the sensitivity to in-plane flexible vibration is low but the sensitivity to in-plane whole body vibration 
is very large as a consequence of attachment of a vertex mirror to the structure of interest. The models originating from the framework offer vibrometer designers and users unprecedented opportunities to optimise configurations, compare systems and enhance data interpretation.

\section{Acknowledgement}

The authors would like to acknowledge support from the Engineering and Physical Sciences Research Council for Mario Tirabassi. 


\section{References}

[1] B. Stoffregen and A. Felske, Scanning laser Doppler vibration analysis system. Trans. SAE Technical Paper Series 850327 (1985 ) 934-940. (DOI 10.4271/850327)

[2] B. Junge, Experiences with scanning laser vibrometry in automotive industries. Proc. 1st Int. Conf on Vibration Measurements by Laser Techniques, (ed. E.P. Tomasini), SPIE vol. 2358 Ancona, Italy, 1994, pp. 377-382.

[3] X.D. Zeng, A.L. Wicks and T.E. Allen, Pose estimation of a scanning laser Doppler vibrometer with applications to the automotive industry. Opt. Eng. 37(5) (1998) 1442-1447. (DOI $10.1117 / 1.601653)$

[4] M.A. Beeck and W. Hentschel, Laser metrology - a diagnostic tool in automotive development processes. Opt. Lasers Eng. 34 (2) (2000) 101-120.(DOI 10.1016/S0143-8166(00)00077-4)

[5] J. Hancox, B.C. Staples and R.J. Parker, The application of scanning laser Doppler vibrometry in aero-engine development. Proc. IMechE - J. Aero. Eng. 209 (1995) 35-42. (DOI 10.1243/PIME_PROC_1995_209_268_02)

[6] Polytec, Structure-borne noise measurement with a robot-controlled 3-D scanning laser vibrometer. InFocus. 1 (2008) 5-7. http://www.polytec.com/no cache/int/news/infocusmagazine $/$ cid $=3280 \&$ did $=3622 \&$ sechash $=5$ bca964d (February 2011)

[7] A.B. Stanbridge and D.J. Ewins, Measurement of translational and angular vibration using a scanning laser Doppler Vibrometer. Shock and Vib. 3(2) (1996) 141-152.

[8] P. Sriram, J.I. Craig and S. Hanagud, A scanning laser Doppler vibrometer for modal testing. Int. J. Analytical and Experimental Modal Analysis 5(3) (1990) 155-167.

[9] A.B. Stanbridge and D.J. Ewins, Modal testing using a scanning laser Doppler vibrometer. Mech. Syst. Signal Process. 13(2) (1999) 255-270. (DOI 10.1006/mssp.1998.1209)

[10] S. Vanlanduit, P. Guillaume and J. Schoukens, Broadband vibration measurements using a continuously scanning laser vibrometer. Meas. Sci. Technol. 13(10) (2002) 1574-1582. (DOI 10.1088/0957-0233/13/10/310)

[11] J.P. La, J. Choi, S.Y. Wang, K. Kim and K. Park, Continuous scanning laser Doppler vibrometer for mode shape analysis. Opt. Eng. 42(3) (2003) 730-737. (DOI 10.1117/1.1533794)

[12] A.B. Stanbridge, M. Martarelli and D.J. Ewins, Measuring area vibration mode shapes with a continuous-scan LDV. Measurement 35(2) (2004) 181-189. (DOI 10.1016/j.measurement.2003.07.005)

[13] M.S. Allen and M.W. Sracic, A new method for processing impact excited continuous-scan laser Doppler vibrometer measurements. Mech. Syst. Signal Process. 24(3) (2010) 721-735. (DOI 10.1016/j.ymssp.2009.11.004)

[14] P. Castellini, and N. Paone, Development of the tracking laser vibrometer: performance and uncertainty analysis. Rev. Sci. Instrum. 71(12) (2000) 4639-4647. (DOI 10.1063/1.1319862)

[15] R.A. Lomenzo, A.J. Barker and A.L. Wicks, Laser vibrometry system for rotating bladed disks. Proc. $17^{\text {th }}$ Int. Modal Analysis Conf. SEM vol. 3727, Kissimmee FL, February 1999 pp. $277-$ 282.

[16] B.J. Halkon and S.J. Rothberg, Vibration measurements using continuous scanning laser vibrometry: advanced aspects in rotor applications. Mech. Syst. Signal Process. 20(6) (2006) 1286-1299. (DOI 10.1016/j.ymssp.2005.11.009).

[17] I.A. Sever, A.B. Stanbridge and D.J. Ewins, Turbomachinery blade vibration measurements with tracking LDV under rotation. Proc.7th Int. Conf. Vibration Measurements by Laser Techniques, (ed. E.P. Tomasini), SPIE Vol 6345, Ancona, Italy, 2006, paper 6345-21. (DOI 10.1117/12.693172)

[18] S. Boedecker, A. Drabenstadt, L. Heller, A. Kraft, A. Leonhardt, C. Pape, S. Ristau, E. Reithmeier and C. Rembe, Optical derotator for scanning vibrometer measurements on rotating 
objects. Proc.7th Int. Conf. Vibration Measurements by Laser Techniques, (ed. E.P. Tomasini), SPIE Vol 6345, Ancona, Italy, 2006, paper 6345-22. (DOI 10.1117/12.693066)

[19] P. Castellini and R. Montanini, Automotive components vibration measurements by tracking laser Doppler vibrometry: advances in signal processing. Meas. Sci. Technol. 13 (2002) 12661279. (DOI 10.1088/0957-0233/13/8/314)

[20] H. Dietzhausen, K. Bendel and N. Scelles, Tracking scanning laser Doppler vibrometers: extending laser vibrometry to arbitrarily moving objects. Proc. IMAC XXI: A Conference \& Exposition on Structural Dynamics, Kissimmee, Florida, 2003, Paper \#168 [CD-ROM].

[21] B.J. Halkon and S.J. Rothberg, Synchronised-scanning laser vibrometry. Proc.6th Int. Conf. Vibration Measurements by Laser Techniques, (ed. E.P. Tomasini), SPIE Vol. 5503, Ancona, Italy, 2004 pp 260-271. (DOI 10.1117/12.579759).

[22] D. Di Maio and D.J. Ewins, CAISER MYMESIS: a new software platform for virtual and actual vibration testing on rotating structures using a continuously scanning LDV technique.

Proc.7th Int. Conf. Vibration Measurements by Laser Techniques, (ed. E.P. Tomasini), SPIE Vol 6345, Ancona, Italy, 2006, paper 6345-26 \{CD-ROM]. (DOI 10.1117/12.693081)

[23] D. Di Maio and D.J. Ewins, Applications of continuous tracking SLDV measurement methods to axially symmetric rotating structures using different excitation methods. Mech. Syst. Signal Process. 24(8) (2010) 3013-3036 (DOI 10.1016/j.ymssp.2010.06.012)

[24] Q.V. Davis and W.K. Kulczyk, Vibrations of turbine blades measured by means of a laser. Nature 222 (1969) 475-476. (DOI 10.1038/222475a0)

[25] R.A. Cookson and P. Bandyopadhyay, A fiber-optic laser-Doppler probe for vibration analysis of rotating machines. Trans. ASME J. Eng. Power 102 (1980) 607-612. (DOI 10.1115/1.3230310)

[26] A.K. Reinhardt, J.R. Kadambi, R.D. Quinn, Laser vibrometry measurements of rotating blade vibrations. Trans. ASME J. Eng. Gas Turbines Power 117 (1995) 484-488. (DOI 10.1115/1.2814121)

[27] A.J. Oberholster and P.S. Heyns, Online condition monitoring of axial-flow turbomachinery blades using rotor axial Eulerian laser Doppler vibrometry. Mech. Syst. Signal Process. 23(5) (2009) 1634-1643. (DOI 10.1016/j.ymssp.2009.01.001)

[28] N.A. Halliwell, The laser torsional vibrometer: a step forward in rotating machinery diagnostics. J. Sound Vib. 190(3) (1996) 399-418. (DOI 10.1006/jsvi.1996.0071)

[29] S.J. Drew and B.J. Stone, Torsional (rotational) vibration: Excitation of small rotating machines. J. Sound Vib. 201(4) (1997) 437-463. (DOI 10.1006/jsvi.1996.0778)

[30] C. Collette and A. Preumont, Laser measurement of torsional vibrations/longitudinal creepage of a railway wheel set on a scaled test bench. Opt. Lasers Eng. 47 (3-4) (2009) 385-389. (DOI 10.1016/j.optlaseng.2008.03.011)

[31] S.J. Rothberg and N.A. Halliwell, Vibration measurements on rotating machinery using laser Doppler velocimetry. Trans. ASME J. Vib. Acoust. 116 (1994) 326-331. (DOI 10.1115/1.2930432)

[32] J.R. Bell and S.J. Rothberg, Laser vibrometers and contacting transducers, target rotation and six degree-of-freedom vibration: what do we really measure? J. Sound Vib. 237(2) (2000) 245261. (DOI 10.1006/jsvi.2000.3053)

[33] J.R. Bell and S.J. Rothberg, Rotational vibration measurements using laser vibrometry: comprehensive theory and practical application. J. Sound Vib. 238(4) (2000) 673-690. (DOI 10.1006/jsvi.2000.3134)

[34] B.J. Halkon and S.J. Rothberg, Rotor vibration measurements using laser Doppler vibrometry; essential post-processing for resolution of radial and pitch / yaw vibrations. Trans. ASME J. Vib. Acoust. 128 (2006) 8-20. (DOI 10.1115/1.2149389)

[35] B.J. Halkon and S.J. Rothberg, Vibration measurements using continuous scanning laser Doppler vibrometry: theoretical velocity sensitivity analysis with applications. Meas. Sci. Technol. 14 (2003) 382-393. (DOI 10.1088/0957-0233/14/3/318) 
[36] M. Tirabassi and S.J. Rothberg, Scanning LDV using wedge prisms. Opt. Lasers Eng. 47(3-4) (2009) 454-460. (DOI 10.1016/j.optlaseng.2008.06.003)

[37] S. Di Battista, D. Di Maio, D.J. Ewins, P. Castellini and E.P. Tomasini, Development of a comprehensive mathematical model for simulating the effects of misalignments in vibration measurements using scanning LDV measurement systems, Proc.9th Int. Conf. Vibration Measurements by Laser and Noncontact Techniques, (ed. E.P. Tomasini), Ancona, Italy, 2010, pp29-41.

[38] J. Oldengarm, A.H. Von Krieken and H. Raterink, Laser Doppler velocimeter with optical frequency shifting. Opt. Laser Technol. 5 (1973) 249-252. (DOI 10.1016/0030-3992(73)900509)

[39] P. Buchave, Laser Doppler velocimeter with variable optical frequency shift. Opt. Laser Technol. 7 (1975) 11-16.

[40] B.M. Watrasiewicz and M.J. Rudd, Laser Doppler measurement. Butterworths, London, 1976.

[41] A.S. Glassner (ed.), An Introduction to Ray Tracing. Academic Press, London, 1989.

[42] H.R. Harrison and T. Nettleton, Advanced Engineering Dynamics. Arnold, London, 1997.

[43] Polytec, Surface vibration measurement on rotating components: non-contact deflection shape analysis on rotating components using the PSV-A-440 optical derotator. Application Note, 2010. http://www.polytec.com/eu/products/vibration-sensors/scanning-vibrometers/psv-a-440optical-derotator/ (February 2011).

[44] I.A. Sever, Experimental validation of turbomachinery blade vibration predictions. $\mathrm{PhD}$ Thesis, Imperial College, London, 2004.

http://www3.imperial.ac.uk/pls/portallive/docs/1/40373698.PDF (February 2011) 


\section{Appendix A: Expansion of vibration vectors in equation (4b)}

Vector expressions for the target's whole body translational vibration velocity, $\overrightarrow{V_{O^{*}}}$, and the associated displacement, $\overrightarrow{O O^{*}}$, can be written in terms of $\mathrm{x}$-, $\mathrm{y}$ - and $\mathrm{z}$-components of translational velocity at $O^{*}, V_{0^{*} x}, V_{0^{*} y}$ and $V_{0^{*} z}$ respectively:

$\overrightarrow{V_{O^{*}}}=\left[\begin{array}{ccc}\hat{x} & \hat{y} & \hat{z}\end{array}\right]\left[\begin{array}{lll}V_{0^{*} x} & V_{0^{*} y} & V_{0^{*} z}\end{array}\right]^{T}$

$\overrightarrow{O O^{*}}=\left[\begin{array}{lll}\hat{x} & \hat{y} & \hat{z}\end{array}\right]\left[\int_{0}^{t} V_{0^{*} x} d t \quad \int_{0}^{t} V_{0^{*} y} d t \quad \int_{0}^{t} V_{0^{*} z} d t\right]^{T}$

The vibration velocity due to target flexibility is written in a similar manner to equation (A.1a):

$\overrightarrow{V_{f}\left(P^{\prime}(t)\right)}=\left[\begin{array}{lll}\hat{x} & \hat{y} & \hat{z}\end{array}\right]\left[V_{f x}\left(P^{\prime}(t)\right) \quad V_{f y}\left(P^{\prime}(t)\right) \quad V_{f z}\left(P^{\prime}(t)\right)\right]^{T}$

Angular velocity $\vec{\omega}$ includes both angular vibration velocities around the three coordinate axes, $\dot{\theta}_{x}$, $\dot{\theta}_{y}$ and $\dot{\theta}_{z}$, and any continuous rotation. Continuous rotation at angular frequency $\Omega_{\mathrm{T}}$ occurs around an axis modified by pitch and yaw vibration displacements and the full expression for angular velocity is written as:

$\vec{\omega}=\left[\begin{array}{lll}\hat{x} & \hat{y} & \hat{z}\end{array}\right]\left[\begin{array}{lll}\dot{\theta}_{x} & \dot{\theta}_{y} & \dot{\theta}_{z}\end{array}\right]^{T}+\Omega_{\mathrm{T}}\left[\begin{array}{lll}\hat{x} & \hat{y} & \hat{z}\end{array}\right]\left[\begin{array}{l}y, \theta_{y}\end{array}\right]\left[\begin{array}{l}x, \theta_{x}\end{array}\right]\left[\begin{array}{lll}0 & 0 & 1\end{array}\right]^{T}$

\section{Appendix B: Misalignment between the optical head and the target}

For this case, the equations presented in sections 3 and 4 can be considered as written in an optical frame of reference with an origin $O_{\text {opt }}$ located in the same $x y$ plane as origin $O$ and unit vectors $\hat{x}_{o p t}$, $\hat{y}_{\text {opt }}$ and $\hat{z}_{\text {opt }}$ defining its axes. This arrangement is shown in figure B1. Transformation of any vectors can be made using the relationship:

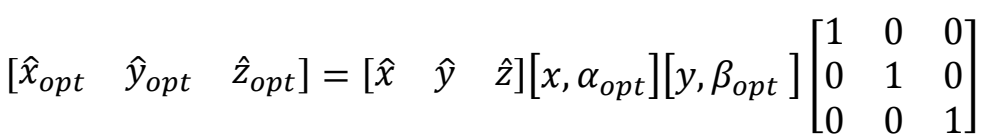

where $\alpha_{\text {opt }}$ and $\beta_{\text {opt }}$ are the misalignment angles, around $x$ - and $y$-axes respectively, between the optical and global coordinate systems. The velocities associated with each deflection point can still be calculated within the optical frame of reference with misalignments incorporated in the same manner. The final beam orientation and the point $T^{\prime}$ in the target plane, however, must be expressed in the 
global system to apply equation (4b). For example, for the Dove prism SLDV, the final beam orientation can be written:

$\hat{b}_{4}=\left[\begin{array}{lll}\hat{x}_{\text {opt }} & \hat{y}_{\text {opt }} & \hat{z}_{\text {opt }}\end{array}\right]\left[\begin{array}{lll}b_{4 x} & b_{4 y} & b_{4 z}\end{array}\right]_{\text {opt }}^{T}=\left[\begin{array}{lll}\hat{x} & \hat{y} & \hat{z}\end{array}\right]\left[x, \alpha_{o p t}\right]\left[\begin{array}{ll}y, \beta_{o p t}\end{array}\right]\left[\begin{array}{lll}b_{4 x} & b_{4 y} & b_{4 z}\end{array}\right]_{\text {opt }}^{T}(\mathrm{~B} .2)$

where $\left[\begin{array}{lll}b_{4 x} & b_{4 y} & b_{4 z}\end{array}\right]_{o p t}^{T}$ is a column vector of the components of $\hat{b}_{4}$ in the optical axes.

Identification of $T^{\prime}$ follows from re-expressing $\overrightarrow{O D^{\prime}}$ in the global axes using:

$\overrightarrow{O D^{\prime}}=\overrightarrow{O O_{o p t}}+\overrightarrow{O_{o p t} D^{\prime}}$

in which $\overrightarrow{O_{o p t} D^{\prime}}$ is found by transforming $\overrightarrow{O D^{\prime}}$ from equation (35d) into the global system using equation (B.1). Equation (B.3) is illustrated in figure B1 including how $\overrightarrow{O O_{o p t}}$ connects the origins of the global and optical systems and expresses translational misalignment:

$\overrightarrow{O O_{o p t}}=\left[\begin{array}{lll}\hat{x} & \hat{y} & \hat{z}\end{array}\right]\left[\begin{array}{lll}\Delta x_{\text {opt }} & \Delta y_{\text {opt }} & 0\end{array}\right]^{T}$

In equation (B.4), $\Delta x_{o p t}$ and $\Delta y_{o p t}$ would be inputs to the model. With $\hat{b}_{4}$ and $\overrightarrow{O D^{\prime}}$ now expressed in the global coordinate system, equation (36) is applied to find $\overrightarrow{O T^{\prime}}$.

\section{Appendix C: Calculation of $\left|\overrightarrow{B^{\prime} C^{\prime}}\right|$ and $\gamma_{\text {con }}$ for equations (47a) and (50b).}

Combining equations (48) and (49c) and equating components (identified by subscripts $x, y$ and $z$ ) leads to the following three equations:

$$
\begin{aligned}
& \left(\overrightarrow{O B^{\prime}}-\overrightarrow{O D^{*}}\right)_{x}+\left|\overrightarrow{B^{\prime} C^{\prime}}\right| \hat{b}_{2 x}=-\frac{\left|\overrightarrow{\mathrm{D}^{*} \mathrm{C}^{\prime}}\right|}{\sqrt{2}}\left(\beta+\sin \gamma_{\mathrm{con}}\right) \\
& \left(\overrightarrow{O B^{\prime}}-\overrightarrow{O D^{*}}\right)_{y}+\left|\overrightarrow{B^{\prime} C^{\prime}}\right| \hat{b}_{2 y}=\frac{\left|\overrightarrow{\mathrm{D}^{*} \mathrm{C}^{\prime}}\right|}{\sqrt{2}}\left(\alpha+\cos \gamma_{\mathrm{con}}\right) \\
& \left(\overrightarrow{O B^{\prime}}-\overrightarrow{O D^{*}}\right)_{z}+\left|\overrightarrow{B^{\prime} C^{\prime}}\right| \hat{b}_{2 z}=\frac{\left|\overrightarrow{\mathrm{D}^{*} \mathrm{C}^{\prime}}\right|}{\sqrt{2}}\left(\beta \sin \gamma_{\mathrm{con}}+\alpha \cos \gamma_{\mathrm{con}}-1\right)
\end{aligned}
$$

The benefit of small angle approximations in equation (49c) is the formulation of a quadratic equation for $\left|\overrightarrow{B^{\prime} C^{\prime}}\right|$. This is derived in three steps. The first is the substitution of equations (C.1a) and (C.1b) into (C.1c) to give: 


$$
\frac{\left|\overrightarrow{D^{*} C^{\prime}}\right|}{\sqrt{2}}=\frac{\left|\overrightarrow{B^{\prime} C^{\prime}}\right|\left(\alpha \hat{b}_{2 y}-\beta \hat{b}_{2 x}-\hat{b}_{2 z}\right)+\left(\alpha\left(\overrightarrow{O B^{\prime}}-\overrightarrow{O D^{*}}\right)_{y}-\beta\left(\overrightarrow{O B^{\prime}}-\overrightarrow{O D^{*}}\right)_{x}-\left(\overrightarrow{O B^{\prime}}-\overrightarrow{O D^{*}}\right)_{z}\right)}{\left(1+\alpha^{2}+\beta^{2}\right)}=a\left|\overrightarrow{B^{\prime} C^{\prime}}\right|+b
$$

The second step is the combination of equations $(\mathrm{C} .1 \mathrm{a} \& \mathrm{~b})$ based on the relationship $\sin ^{2} \gamma_{\text {con }}+\cos ^{2} \gamma_{\text {con }}=1$ to give:

$0.5\left|\overrightarrow{\mathrm{D}^{*} \mathrm{C}^{\prime}}\right|^{2}\left(\alpha^{2}+\beta^{2}-1\right)$

$+\sqrt{2}\left|\overrightarrow{\mathrm{D}^{*} C^{\prime}}\right|\left(\beta\left(\left(\overrightarrow{O B^{\prime}}-\overrightarrow{O D^{*}}\right)_{x}+\left|\overrightarrow{B^{\prime} C^{\prime}}\right| \hat{b}_{2 x}\right)-\alpha\left(\left(\overrightarrow{O B^{\prime}}-\overrightarrow{O D^{*}}\right)_{y}+\left|\overrightarrow{B^{\prime} C^{\prime}}\right| \hat{b}_{2 y}\right)\right)$

$+\left(\left(\overrightarrow{O B^{\prime}}-\overrightarrow{O D^{*}}\right)_{x}+\left|\overrightarrow{B^{\prime} C^{\prime}}\right| \hat{b}_{2 x}\right)^{2}+\left(\left(\overrightarrow{O B^{\prime}}-\overrightarrow{O D^{*}}\right)_{y}+\left|\overrightarrow{B^{\prime} C^{\prime}}\right| \hat{b}_{2 y}\right)^{2}=0$

Eliminating $\left|\overrightarrow{\mathrm{D}^{*} \mathrm{C}^{\prime}}\right|$ between equations (C.2) and (C.3) is the third step, leading to the quadratic equation in $\left|\overrightarrow{B^{\prime} C^{\prime}}\right|$ :

$$
\begin{aligned}
& 0=\left|\overrightarrow{B^{\prime} C^{\prime}}\right|^{2}\left(a^{2}\left(1-\alpha^{2}-\beta^{2}\right)-2 a\left(\beta \hat{b}_{2 x}-\alpha \hat{b}_{2 y}\right)-\hat{b}_{2 x}^{2}-\hat{b}_{2 y}^{2}\right) \\
& +2\left|\overrightarrow{B^{\prime} C^{\prime}}\right|\left(a b\left(1-\alpha^{2}-\beta^{2}\right)+a\left(\alpha\left(\overrightarrow{O B^{\prime}}-\overrightarrow{O D^{*}}\right)_{y}-\beta\left(\overrightarrow{O B^{\prime}}-\overrightarrow{O D^{*}}\right)_{x}\right)+b\left(\alpha \hat{b}_{2 y}-\beta \hat{b}_{2 x}\right)\right. \\
& \left.\quad-\hat{b}_{2 x}\left(\overrightarrow{O B^{\prime}}-\overrightarrow{O D^{*}}\right)_{x}-\hat{b}_{2 y}\left(\overrightarrow{O B^{\prime}}-\overrightarrow{O D^{*}}\right)_{y}\right) \\
& +b^{2}\left(1-\alpha^{2}-\beta^{2}\right)+2 b\left(\alpha\left(\overrightarrow{O B^{\prime}}-\overrightarrow{O D^{*}}\right)_{y}-\beta\left(\overrightarrow{O B^{\prime}}-\overrightarrow{O D^{*}}\right)_{x}\right)-\left(\overrightarrow{O B^{\prime}}-\overrightarrow{O D^{*}}\right)_{x}^{2}-\left(\overrightarrow{O B^{\prime}}-\overrightarrow{O D^{*}}\right)_{y}^{2}
\end{aligned}
$$

The negative root gives a positive value for $\left|\overrightarrow{B^{\prime} C^{\prime}}\right|$ and is selected. The point of incidence on the conical mirror can now be found from equation (47a). Finally, having found $\left|\overrightarrow{B^{\prime} C^{\prime}}\right|$, formulation of $\tan \gamma_{c o n}$ is possible by rearrangement of equations (C.1a\&b) and substitution of equation (C.2):

$$
\tan \gamma_{c o n}=\frac{-\left(\left(\overrightarrow{O B^{\prime}}-\overrightarrow{O D^{*}}\right)_{x}+\left|\overrightarrow{B^{\prime} C^{\prime}}\right| \hat{b}_{2 x}\right)-\beta\left(a\left|\overrightarrow{B^{\prime} C^{\prime}}\right|+b\right)}{\left(\left(\overrightarrow{O B^{\prime}}-\overrightarrow{O D^{*}}\right)_{y}+\left|\overrightarrow{B^{\prime} C^{\prime}}\right| \hat{b}_{2 y}\right)-\alpha\left(a\left|\overrightarrow{B^{\prime} C^{\prime}}\right|+b\right)}
$$

This enables identification of the surface normal at the point of incidence on the conical mirror according to equation (50b).

\section{Appendix D: Parameters for multiple simulations contributing to Tables 1-4}

\section{D1: Dual mirrors SLDV}




\section{Misalignments:}

$\beta_{L}$ from $-0.4^{\mathrm{O}}$ to $0.4^{\mathrm{O}}$ in steps of $0.2^{\mathrm{O}}$ and $\gamma_{L}$ from $-0.1^{\mathrm{O}}$ to $0.1^{\mathrm{O}}$ in steps of $0.1^{\mathrm{O}}$.

$\Delta y_{A}$ from $-2 \mathrm{~mm}$ to $2 \mathrm{~mm}$ in steps of $2 \mathrm{~mm}$ and $\Delta z_{A}$ from $-10 \mathrm{~mm}$ to $10 \mathrm{~mm}$ in steps of $5 \mathrm{~mm}$.

$\alpha_{1}$ and $\gamma_{2}$ from $-0.1^{\mathrm{O}}$ to $0.1^{\mathrm{O}}$ in steps of $0.1^{\mathrm{O}}$.

$\beta_{1}$ and $\beta_{2}$ from $-0.25^{\circ}$ to $0.25^{\circ}$ in steps of $0.25^{\circ}$.

$\Delta x_{B}, \Delta y_{B}, \Delta y_{C}$ and $\Delta z_{C}$ from $-3 \mathrm{~mm}$ to $3 \mathrm{~mm}$ in steps of $3 \mathrm{~mm}, \Delta z_{B}=\Delta x_{C}=0$.

Geometry for all simulations: $x_{A}=0.2 \mathrm{~m}, d_{S}=50 \mathrm{~mm}, z_{A}=1.2 \mathrm{~m}$.

\section{D2: Dove prism SLDV}

Misalignments:

$\alpha_{L}$ from $-0.1^{\mathrm{O}}$ to $0.1^{\mathrm{O}}$ in steps of $0.1^{\mathrm{O}}$ and $\beta_{L}$ from $-0.6^{\mathrm{O}}$ to $0.6^{\mathrm{O}}$ in steps of $0.2^{\mathrm{O}}$.

$\Delta x_{A}$ from $-12 \mathrm{~mm}$ to $12 \mathrm{~mm}$ in steps of $4 \mathrm{~mm}$ and $\Delta y_{A}$ from $-6 \mathrm{~mm}$ to $6 \mathrm{~mm}$ in steps of $3 \mathrm{~mm}$.

$\alpha_{1}$ from $-0.1^{\mathrm{O}}$ to $0.1^{\mathrm{O}}$ in steps of $0.1^{\mathrm{O}}$ and $\beta_{1}$ from $-0.6^{\mathrm{O}}$ to $0.6^{\mathrm{O}}$ in steps of $0.2^{\mathrm{O}}$.

$\Delta x_{B}$ from $-12 \mathrm{~mm}$ to $12 \mathrm{~mm}$ in steps of $4 \mathrm{~mm}$ and $\Delta y_{B}$ from $-4 \mathrm{~mm}$ to $4 \mathrm{~mm}$ in steps of $2 \mathrm{~mm}, \Delta z_{B}=0$.

Geometry for all simulations:

$z_{A}=1.4 \mathrm{~m}, z_{B}=1.2 \mathrm{~m},|\overrightarrow{B D}|=6.6 \mathrm{~cm}, h=1 \mathrm{~cm}, \alpha_{L 0}=2.0875^{\circ}, \beta_{L 0}=0^{\circ}$.

Refractive indexes: 1 for air $\left(\varepsilon_{0}\right)$ and 1.5 for the Dove prism $\left(\varepsilon_{1}\right)$.

\section{D3: Self-tracking LDV}

Misalignments for Table 3:

$\alpha_{L}$ from $-0.1^{\mathrm{O}}$ to $0.1^{\mathrm{O}}$ in steps of $0.1^{\mathrm{O}}$ and $\beta_{L}$ from $-0.4^{\mathrm{O}}$ to $0.4^{\mathrm{O}}$ in steps of $0.2^{\mathrm{O}}$.

$\Delta x_{A}$ from $-1 \mathrm{~mm}$ to $1 \mathrm{~mm}$ in steps of $1 \mathrm{~mm}$ and $\Delta y_{A}$ from $-0.5 \mathrm{~mm}$ to $0.5 \mathrm{~mm}$ in steps of $0.5 \mathrm{~mm}$.

$\alpha_{v}$ from $-0.2^{\mathrm{O}}$ to $0.2^{\mathrm{O}}$ in steps of $0.2^{\mathrm{O}}$ and $\beta_{v}$ from $-0.2^{\mathrm{O}}$ to $0.2^{\mathrm{O}}$ in steps of $0.2^{\mathrm{O}}$.

$\Delta z_{B}$ from $-1 \mathrm{~mm}$ to $1 \mathrm{~mm}$ in steps of $1 \mathrm{~mm}$.

$\alpha_{2}$ from $-0.2^{\mathrm{O}}$ to $0.2^{\mathrm{O}}$ in steps of $0.2^{\mathrm{O}}$ and $\beta_{2}$ from $-0.4^{\mathrm{O}}$ to $0.4^{\mathrm{O}}$ in steps of $0.2^{\mathrm{O}}$.

$\Delta x_{D}, \Delta y_{D}$ and $\Delta z_{D}$ from $-3 \mathrm{~mm}$ to $3 \mathrm{~mm}$ in steps of $3 \mathrm{~mm}$.

Misalignments for Table 4:

$\beta_{L}$ reduced to $-0.2^{\mathrm{O}}$ to $0.2^{\mathrm{O}}$ in steps of $0.2^{\mathrm{O}}$.

$\alpha_{v}$ reduced to $-0.1^{\mathrm{O}}$ to $0.1^{\mathrm{O}}$ in steps of $0.1^{\mathrm{O}}$ and $\beta_{v}$ from $-0.1^{\mathrm{O}}$ to $0.1^{\mathrm{O}}$ in steps of $0.1^{\mathrm{O}}$.

$\beta_{2}$ reduced to $-0.2^{\mathrm{O}}$ to $0.2^{\mathrm{O}}$ in steps of $0.2^{\mathrm{O}}$.

$\Delta x_{D}, \Delta y_{D}$ and $\Delta z_{D}$ reduced to $-2 \mathrm{~mm}$ to $2 \mathrm{~mm}$ in steps of $2 \mathrm{~mm}$.

Geometry for all simulations:

$z_{B}=0.05 \mathrm{~m}$; cone angle $=45^{\circ}$, vertex mirror angle $=-45^{\circ}, z_{A}=z_{D}+0.2 \mathrm{~m}$. 


\section{Appendix E: Unequal amplitude sidebands due to in-plane vibration}

Peaks of unequal amplitude at frequencies $\omega_{x} \pm \Omega_{T}$ appear as a consequence of in-plane vibration $V_{O x} \hat{x}$. An approximate expression for beam orientation can be used in conjunction with equation (4c) for the case of in-plane vibration (only) of a rotating target:

$\left.-\hat{b}_{3} \cdot \overrightarrow{V_{T^{\prime}}} \approx-\left(-a_{1} \sin \Omega_{\mathrm{s}} \mathrm{t} \hat{x}+a_{1} \cos \Omega_{\mathrm{s}} \mathrm{t} \hat{y}-a_{2} \hat{z}\right) \cdot\left(V_{O^{*} x} \hat{x}+\Omega_{\mathrm{T}} \hat{z} \times \overrightarrow{O T^{\prime}}-\overrightarrow{O O^{*}}\right]\right)$

where $a_{1}$ is typically small (dependent on scan radius), $a_{2}$ is close to one and $\Omega_{\mathrm{s}}$ is the scan frequency. The in-plane velocity and its associated in-plane displacement can be written:

$V_{O^{*} x} \hat{x}=\left(v_{O^{*} x} \sin \omega_{x} t\right) \hat{x}$

$\overrightarrow{O O^{*}}=\left(-\frac{v_{O^{*} x}}{\omega_{x}} \cos \omega_{x} t\right) \hat{x}$

while the circular scan path can reasonably be approximated as:

$\overrightarrow{O T^{\prime}} \approx\left(-r_{s} \sin \Omega_{\mathrm{s}} \mathrm{t}\right) \hat{x}+\left(r_{s} \cos \Omega_{\mathrm{s}} \mathrm{t}\right) \hat{y}$

Substitution of equations (D.2a\&b) and (D.3) into the expression for $\overrightarrow{V_{T^{\prime}}}$ in equation (D.1) results in:

$\overrightarrow{V_{T^{\prime}}} \approx\left(v_{O^{*} x} \sin \omega_{x} t-\Omega_{\mathrm{T}} r_{s} \cos \Omega_{\mathrm{s}} \mathrm{t}\right) \hat{x}+\Omega_{\mathrm{T}}\left(-r_{s} \sin \Omega_{\mathrm{s}} \mathrm{t}+\frac{v_{O^{*} x}}{\omega_{x}} \cos \omega_{x} t\right) \hat{y}$

from which the dot product can be formulated:

$-\hat{b}_{3} \cdot \overrightarrow{V_{T^{\prime}}} \approx a_{1} v_{O^{*} x}\left(\sin \Omega_{s} t \sin \omega_{x} t-\frac{\Omega_{T}}{\omega_{x}} \cos \Omega_{s} t \cos \omega_{x} t\right)$

This expression shows a product of sines that is dependent on scanning and in-plane vibration but not target rotation followed by a product of cosines that is additionally dependent on target rotation. On expansion, each product is seen to contribute equal amplitude components at sum and difference frequencies:

$-\hat{b}_{3} \cdot \overrightarrow{V_{T^{\prime}}} \approx \frac{a_{1} v_{O^{*} x}}{2}\left[\left(\cos \left(\omega_{x}-\Omega_{s}\right) t-\cos \left(\omega_{x}+\Omega_{s}\right) t\right)-\frac{\Omega_{T}}{\omega_{x}}\left(\cos \left(\omega_{x}-\Omega_{s}\right) t+\cos \left(\omega_{x}+\Omega_{s}\right) t\right)\right]$ (D.6)

It is the relative phasing of the sum and difference frequency components and the equivalence between $\Omega_{\mathrm{S}}$ and $\Omega_{\mathrm{T}}$ for tracking that results in the peaks at frequencies $\omega_{x} \pm \Omega_{T}$ with a ratio of amplitudes equal to the ratio of the frequencies themselves: 
$-\hat{b}_{3} \cdot \overrightarrow{V_{T^{\prime}}} \approx \frac{a_{1} v_{O^{*} x}}{2}\left[\left(1-\frac{\Omega_{T}}{\omega_{x}}\right) \cos \left(\omega_{x}-\Omega_{T}\right) t-\left(1+\frac{\Omega_{T}}{\omega_{x}}\right) \cos \left(\omega_{x}+\Omega_{T}\right) t\right]$

While the amplitude of each peak is clearly dependent on vibration frequency, the mean amplitude of these peaks is not. The same effect occurs in the self-tracking system where the vertex mirror produces a sensitivity to whole body in-plane vibration according to:

$\left(\hat{b}_{2}-\hat{b}_{1}\right) \cdot \overrightarrow{V_{B^{\prime}}}=\left(\hat{b}_{2}-\hat{b}_{1}\right) \cdot\left(V_{O^{*} x} \hat{x}+\Omega_{\mathrm{T}} \hat{z} \times\left(\overrightarrow{O B^{\prime}}-\overrightarrow{O O^{*}}\right)\right) \approx \hat{b}_{2} \cdot\left(V_{O^{*} x} \hat{x}-\Omega_{\mathrm{T}} \hat{z} \times \overrightarrow{O O^{*}}\right)$

where the simplification results from $\hat{b}_{1}$ and $\overrightarrow{O B^{\prime}}$ having such dominant $z$-components (without misalignment they have only $z$-components). Equation (D.8) has the same pattern of one term, $\left(\hat{b}_{2} \cdot V_{O x} \hat{x}\right)$, that is dependent on scanning and in-plane vibration but not target rotation followed by a second term, $\hat{b}_{2} \cdot\left(\Omega_{\mathrm{T}} \hat{Z} \times \overrightarrow{O O^{*}}\right)$, that is additionally dependent on target rotation. Here much larger amplitudes result because $\hat{b}_{2}$ has sinusoidal components in $x$ - and $y$-directions of amplitude close to 1 . 


\section{Figure captions}

Figure 1 - Reflection and refraction of a laser beam at $P^{\prime}$

Figure 2: Dual mirror SLDV: a) full geometry without misalignment b) with misalignments of the laser source and mirrors.

Figure 3: Dove prism SLDV: a) full geometry with beam angular alignment for scanning (zero misalignment) and b) with misalignments of the laser source and prism.

Figure 4: Self-tracking LDV: a) full geometry without misalignment and b) with misalignments of the laser source, vertex mirror and conical mirror.

Figure 5a: Orientation of $\hat{n}_{C^{\prime}}$, the surface normal unit vector on the conical mirror: (1) an initial orientation in the $-z_{c o n}$-direction, (2) a negative rotation by the cone angle ( $\pi / 4 \mathrm{rad}$ ) around $x_{\text {con }}$, (3) rotation around $z_{c o n}$ by the angle $\gamma_{c o n}$. Relocating the vectors at (2) to (2a) and (3) to (3a) shows how the tip of the surface normal unit vector follows a circular path as the laser beam scans around the mirror.

Figure 5b: Orientation of a unit vector aligned with $\overrightarrow{D^{*} C^{\prime}}$. (1) initial orientation in the $-\hat{z}_{c o n}$-direction, (2) rotation by the cone angle $(\pi / 4 \mathrm{rad})$ around $\hat{x}_{c o n}$, (3) rotation around $\hat{z}_{c o n}$ by the angle $\gamma_{c o n} .\left(\gamma_{c o n}\right.$ rotation appears clockwise as viewed from the right of the figure).

Figure 5c: Orientation of conical mirror axes relative to cone axes: (1) $\gamma_{c o n}=0, y_{c m}$ and $z_{c m}$ lie in $y_{c o n} z_{c o n}$ plane, (2) $\gamma_{c o n}=\pi / 4$ rads, $y_{c m}$ and $z_{c m}$ lie in $x_{c o n} z_{c o n}$ plane.

Figure 6: Typical velocities in the dual mirror SLDV in the presence of misalignments $\left(\beta_{L}=0.25^{\circ}\right.$, $\gamma_{L}=-0.1^{\circ}, \Delta y_{A}=2 \mathrm{~mm}, \Delta z_{A}=-10 \mathrm{~mm}, \alpha_{1}=0.1^{\circ}, \beta_{1}=-0.1^{\circ}, \Delta x_{B}=2 \mathrm{~mm}, \Delta y_{B}=-2 \mathrm{~mm}$, $\left.\beta_{2}=-0.1^{\circ}, \gamma_{2}=0.1^{\circ}, \Delta y_{C}=5 \mathrm{~mm}, \Delta z_{C}=-5 \mathrm{~mm}\right)$ and sinusoidal target vibrations $\left(\overrightarrow{V_{f z}}\right.$ of amplitude $1 \mathrm{~mm} / \mathrm{s} / \mathrm{rad} / \mathrm{s}$ at $8 \Omega_{\mathrm{T}}$ and $\overrightarrow{V_{O^{*} x}}$ of amplitude $1 \mathrm{~mm} / \mathrm{s} / \mathrm{rad} / \mathrm{s}$ at $16 \Omega_{\mathrm{T}}$ ):

a) the individual and combined effects of the deflection points on the mirrors, and

b) the combined effects of the mirrors, the effect of the target and total measured velocity.

Figure 7: Typical velocities in the Dove prism SLDV in the presence of misalignments $\left(\alpha_{L}=0.1^{\circ}\right.$, $\beta_{L}=-0.5^{\circ}, \Delta x_{A}=10 \mathrm{~mm}, \Delta y_{A}=-10 \mathrm{~mm}, \alpha_{1}=0.1^{\circ}, \beta_{1}=0.1^{\circ}, \Delta x_{B}=3 \mathrm{~mm}, \Delta y_{B}=-3 \mathrm{~mm}$, $\left.\Delta z_{B}=5 \mathrm{~mm}\right)$ and sinusoidal target vibrations $\left(\overrightarrow{V_{f Z}}\right.$ of amplitude $1 \mathrm{~mm} / \mathrm{s} / \mathrm{rad} / \mathrm{s}$ at $8 \Omega_{\mathrm{T}}$ and $\overrightarrow{V_{O^{*} x}}$ of amplitude $1 \mathrm{~mm} / \mathrm{s} / \mathrm{rad} / \mathrm{s}$ at $16 \Omega_{\mathrm{T}}$ ):

a) the individual and combined effects of the deflection points on the Dove prism, and

b) the combined effects of the prism, the effect of the target and total measured velocity.

Figure 8: Typical velocities in the self-tracking LDV system:

a) in the presence of misalignments $\left(\alpha_{L}=-0.05^{\circ}, \beta_{L}=0.1^{\circ}, \Delta x_{A}=2 \mathrm{~mm}, \Delta y_{A}=-1.5 \mathrm{~mm}\right.$, $\Delta z_{A}=-1.5 \mathrm{~mm}, \alpha_{1}=0.1^{\circ}, \beta_{1}=-0.1^{\circ}, \Delta z_{B}=1 \mathrm{~mm}, \alpha_{2}=0.1^{\circ}, \beta_{2}=-0.3^{\circ}, \Delta x_{D}=-2 \mathrm{~mm}$, $\left.\Delta y_{D}=2 \mathrm{~mm}, \Delta z_{D}=2 \mathrm{~mm}\right)$ and sinusoidal target vibrations $\left(\overrightarrow{V_{f z}}\right.$ of amplitude $1 \mathrm{~mm} / \mathrm{s} / \mathrm{rad} / \mathrm{s}$ at $10 \Omega_{\mathrm{T}}$ and $\overrightarrow{V_{O^{*} x}}$ of amplitude $1 \mathrm{~mm} / \mathrm{s} / \mathrm{rad} / \mathrm{s}$ at $20 \Omega_{\mathrm{T}}$ ) 
b) a surface profile on the conical mirror causing a mean angular deviation in the surface normal of $0.1^{\mathrm{O}}$ (no other misalignments, no vibration).

c) a surface profile as in Fig. $8 \mathrm{~b}$ in the presence of misalignments and vibrations as in Fig. 8a.

Figure B1: Misalignments between global and optical axes.

\section{Table Captions}

Table 1: Mean (standard deviation) of $\mathrm{rms}$ measured velocities $(\mu \mathrm{m} / \mathrm{s} / \mathrm{rad} / \mathrm{s})$ associated with system configuration and typical misalignments.

Table 2: Mean sensitivities (and standard deviations), expressed as the percentage of out-of-plane flexible vibration (at $\omega_{\mathrm{z}}=8 \Omega_{\mathrm{T}}$ ).

Table 3: Mean sensitivities (and standard deviations), expressed as percentage of in-plane whole body vibration (at $\omega_{\mathrm{x}}=16 \Omega_{\mathrm{T}}$ ).

Table 4: Effect of conical mirror surface profile on the mean (standard deviation) of rms measured velocities $(\mu \mathrm{m} / \mathrm{s} / \mathrm{rad} / \mathrm{s})$ for the self-tracking system. 35443 misaligned configurations from 885735 considered. 
Table 1: Mean (standard deviation) of $\mathrm{rms}$ measured velocities $(\mu \mathrm{m} / \mathrm{s} / \mathrm{rad} / \mathrm{s})$ associated with system configuration and typical misalignments.

\begin{tabular}{|c|c|c|c|c|c|c|c|c|}
\hline \multirow{2}{*}{$\begin{array}{l}\text { SLDV } \\
\text { System }\end{array}$} & \multirow{2}{*}{$\begin{array}{l}\text { Misaligned } \\
\text { configurations } \\
\text { used / considered }\end{array}$} & \multicolumn{7}{|c|}{ Order } \\
\hline & & $\mathrm{DC}$ & $0.5 x$ & $1 x$ & $1.5 \mathrm{x}$ & $2 \mathrm{x}$ & $3 x$ & $4 x$ \\
\hline \multirow{2}{*}{$\begin{array}{l}\text { Dual } \\
\text { mirror }\end{array}$} & No misalignments & $\begin{array}{c}\text { No } \\
\text { peak }\end{array}$ & No peak & $\begin{array}{c}\text { No } \\
\text { peak }\end{array}$ & No peak & 29.4 & No peak & $2.63 \mathrm{e}-4$ \\
\hline & $50621 / 1476225$ & $\begin{array}{c}5.19 \\
(4.56)\end{array}$ & No peak & $\begin{array}{c}284 \\
(130)\end{array}$ & No peak & $\begin{array}{c}29.4 \\
(2.02)\end{array}$ & $\begin{array}{c}8.33 \mathrm{e}-2 \\
(5.19 \mathrm{e}-2)\end{array}$ & $\begin{array}{c}2.63 \mathrm{e}-4 \\
(1.50 \mathrm{e}-5)\end{array}$ \\
\hline \multirow{2}{*}{$\begin{array}{l}\text { Dove } \\
\text { prism }\end{array}$} & No misalignments & $\begin{array}{c}\text { No } \\
\text { peak }\end{array}$ & 0.170 & 3.85 & $3.01 \mathrm{e}-2$ & $7.84 \mathrm{e}-5$ & $1.67 \mathrm{e}-8$ & No peak \\
\hline & 15155 / 540225 & $\begin{array}{c}8.72 \\
(7.89)\end{array}$ & $\begin{array}{c}0.219 \\
(4.17 \mathrm{e}-2)\end{array}$ & $\begin{array}{c}221 \\
(113)\end{array}$ & $\begin{array}{c}6.77 \mathrm{e}-2 \\
(2.87 \mathrm{e}-2)\end{array}$ & $\begin{array}{c}4.55 \mathrm{e}-4 \\
(2.65 \mathrm{e}-4)\end{array}$ & $\begin{array}{c}3.08 \mathrm{e}-8 \\
(1.34 \mathrm{e}-8)\end{array}$ & No peak \\
\hline \multirow{3}{*}{$\begin{array}{l}\text { Self- } \\
\text { tracking }\end{array}$} & No misalignments & $\begin{array}{c}\text { No } \\
\text { peak }\end{array}$ & No peak & $\begin{array}{c}\text { No } \\
\text { peak }\end{array}$ & No peak & No peak & No peak & No peak \\
\hline & $\begin{array}{l}\text { Simulation 1: } \\
180675 \text { / } 1476225\end{array}$ & $\begin{array}{c}13.7 \\
(14.7)\end{array}$ & No peak & $\begin{array}{l}1230 \\
(853)\end{array}$ & No peak & $\begin{array}{c}\mathbf{5 5 . 1} \\
(\mathbf{5 7 . 7})\end{array}$ & $\begin{array}{c}3.57 \\
(4.66)\end{array}$ & $\begin{array}{c}0.210 \\
(0.344)\end{array}$ \\
\hline & $\begin{array}{l}\text { Simulation 2: } \\
180675 \text { / } 1476225\end{array}$ & $\begin{array}{c}13.8 \\
(14.7)\end{array}$ & No peak & $\begin{array}{l}1231 \\
(849)\end{array}$ & No peak & $\begin{array}{c}80 \\
(40)\end{array}$ & $\begin{array}{l}40 \\
(4)\end{array}$ & $\begin{array}{c}30 \\
(0.1)\end{array}$ \\
\hline
\end{tabular}


Table 2: Mean sensitivities (and standard deviations), expressed as the percentage of out-of-plane flexible vibration $\left(\right.$ at $\left.\omega_{\mathrm{z}}=8 \Omega_{\mathrm{T}}\right)$.

\begin{tabular}{|c|c|c|c|c|}
\hline \multirow{2}{*}{ SLDV System } & \multirow{2}{*}{$\begin{array}{l}\text { Misaligned } \\
\text { configurations } \\
\text { used / considered }\end{array}$} & \multicolumn{3}{|c|}{ Frequency } \\
\hline & & $\omega_{\mathrm{z}}$ & $\omega_{\mathrm{z}} \pm \Omega_{\mathrm{T}}$ & $\omega_{\mathrm{z}} \pm 2 \Omega_{\mathrm{T}}$ \\
\hline \multirow{2}{*}{ Dual mirror } & No misalignments & 99.9 & No peak & $1.70 \mathrm{e}-3$ \\
\hline & $50621 / 1476225$ & $99.9(1.51 e-3)$ & $1.03 e-2(5.26 e-3)$ & $1.71 \mathrm{e}-3(1.31 \mathrm{e}-5)$ \\
\hline \multirow{2}{*}{ Dove prism } & No misalignments & 99.9 & No peak & No peak \\
\hline & 15155 / 540225 & $99.9(9.88 e-3)$ & $1.19 \mathrm{e}-2(6.52 \mathrm{e}-2)$ & No peak \\
\hline \multirow{3}{*}{ Self-tracking } & No misalignments & 100 & No peak & No peak \\
\hline & $\begin{array}{l}\text { Simulation 1: } \\
180675 \text { / } 1476225\end{array}$ & $99.9(0.106)$ & $3.09 e-3(4.07 e-3)$ & $5.29 \mathrm{e}-2(5.22 \mathrm{e}-2)$ \\
\hline & $\begin{array}{l}\text { Simulation 2: } \\
180675 \text { / } 1476225\end{array}$ & $99.9(0.107)$ & $\begin{array}{l}\text {-ve: } 9 \mathrm{e}-2(9 \mathrm{e}-3) \\
+ \text { +ve: } 2 \mathrm{e}-2(4 \mathrm{e}-3)\end{array}$ & $\begin{array}{r}\text {-ve: } 0.2(4 \mathrm{e}-2) \\
+ \text { +ve: } 5.50 \mathrm{e}-2(4.44 \mathrm{e}-2)\end{array}$ \\
\hline
\end{tabular}


Table 3: Mean sensitivities (and standard deviations), expressed as percentage of in-plane whole body vibration $\left(\right.$ at $\left.\omega_{\mathrm{x}}=16 \Omega_{\mathrm{T}}\right)$.

\begin{tabular}{|c|c|c|c|c|c|c|}
\hline \multirow{2}{*}{$\begin{array}{l}\text { SLDV } \\
\text { System }\end{array}$} & \multirow{2}{*}{$\begin{array}{l}\text { Misaligned } \\
\text { configurations } \\
\text { used / considered }\end{array}$} & \multicolumn{5}{|c|}{ Frequency } \\
\hline & & $\omega_{\mathrm{x}}-2 \Omega_{\mathrm{T}}$ & $\omega_{\mathrm{x}}-\Omega_{\mathrm{T}}$ & $\omega_{\mathrm{x}}$ & $\omega_{\mathrm{x}}+\Omega_{\mathrm{T}}$ & $\omega_{\mathrm{x}}+2 \Omega_{\mathrm{T}}$ \\
\hline \multirow{2}{*}{$\begin{array}{l}\text { Dual } \\
\text { mirror }\end{array}$} & No misalignments & No peak & 1.87 & No peak & 2.13 & No peak \\
\hline & $50621 / 1476225$ & $\begin{array}{c}3.02 \mathrm{e}-5 \\
(2.10 \mathrm{e}-5)\end{array}$ & $\begin{array}{c}1.87 \\
(4.15 e-5)\end{array}$ & $\begin{array}{c}0.240 \\
(0.171)\end{array}$ & $\begin{array}{c}2.13 \\
(4.32 e-5)\end{array}$ & $\begin{array}{c}3.77 \mathrm{e}-5 \\
(2.74 \mathrm{e}-5)\end{array}$ \\
\hline \multirow{2}{*}{$\begin{array}{l}\text { Dove } \\
\text { prism }\end{array}$} & No misalignments & No peak & 1.71 & No peak & 1.94 & No peak \\
\hline & 15155 / 540225 & No peak & $\begin{array}{c}1.76 \\
(0.113)\end{array}$ & $\begin{array}{c}\mathbf{0 . 5 9 8} \\
(\mathbf{0 . 3 5 8})\end{array}$ & $\begin{array}{c}2.00 \\
(0.129)\end{array}$ & No peak \\
\hline \multirow{3}{*}{$\begin{array}{l}\text { Self- } \\
\text { tracking }\end{array}$} & No misalignments & No peak & 46.9 & No peak & 53.1 & No peak \\
\hline & $\begin{array}{l}\text { Simulation 1: } \\
180675 / 1476225\end{array}$ & $\begin{array}{c}1.59 \\
(0.891)\end{array}$ & $\begin{array}{c}46.8 \\
(9.51 \mathrm{e}-2)\end{array}$ & $\begin{array}{c}2.06 \\
(1.45)\end{array}$ & $\begin{array}{c}53.0 \\
(0.111)\end{array}$ & $\begin{array}{c}1.80 \\
(1.01)\end{array}$ \\
\hline & $\begin{array}{l}\text { Simulation 2: } \\
180675 \text { / } 1476225\end{array}$ & $\begin{array}{c}1.59 \\
(0.889)\end{array}$ & $\begin{array}{c}46.9 \\
(9.54 \mathrm{e}-2)\end{array}$ & $\begin{array}{c}2.05 \\
(1.45)\end{array}$ & $\begin{array}{c}53.1 \\
(0.112)\end{array}$ & $\begin{array}{c}1.80 \\
(1.00)\end{array}$ \\
\hline
\end{tabular}


Table 4: Effect of conical mirror surface profile on the mean (standard deviation) of rms measured velocities $(\mu \mathrm{m} / \mathrm{s} / \mathrm{rad} / \mathrm{s})$ for the self-tracking system. 19065 misaligned configurations used from 531441 considered.

\begin{tabular}{|c|c|c|c|c|c|}
\hline \multirow{2}{*}{ Conical mirror surface profile } & \multicolumn{5}{|c|}{ Order } \\
\cline { 2 - 7 } & DC & $1 \mathrm{x}$ & $2 \mathrm{x}$ & $3 \mathrm{x}$ & $4 \mathrm{x}$ \\
\hline Smooth & $\mathbf{4 . 3 4}$ & $\mathbf{1 5 1 3}$ & $\mathbf{1 5 . 5}$ & $\mathbf{0 . 2 0 2}$ & $2.39 \mathrm{e}-3$ \\
& $\mathbf{( 3 . 8 1 )}$ & $\mathbf{( 7 3 1 )}$ & $\mathbf{( 1 0 . 1 )}$ & $\mathbf{( 0 . 2 0 5})$ & $(3.03 \mathrm{e}-3)$ \\
\hline Components at all integer orders & $\mathbf{4 . 8 3}$ & $\mathbf{1 5 3 0}$ & $\mathbf{2 0 0}$ & $\mathbf{2 0 0}$ & $\mathbf{1 0 0}$ \\
with overall Gaussian profile & $\mathbf{( 4 . 1 4 )}$ & $\mathbf{( 7 3 3 )}$ & $\mathbf{( 1 0 )}$ & $\mathbf{( 1 )}$ & $\mathbf{( 0 . 8 )}$ \\
\hline As above but components & $\mathbf{4 . 4 6}$ & $\mathbf{1 5 1 7}$ & $\mathbf{3 0 0}$ & $\mathbf{8 0}$ & $\mathbf{2 0 0}$ \\
at 2x and integer multiples dominate. & $\mathbf{( 3 . 8 7 )}$ & $\mathbf{( 7 2 9 )}$ & $\mathbf{( 1 0 )}$ & $\mathbf{( 1 )}$ & $\mathbf{( 0 . 8 )}$ \\
\hline
\end{tabular}




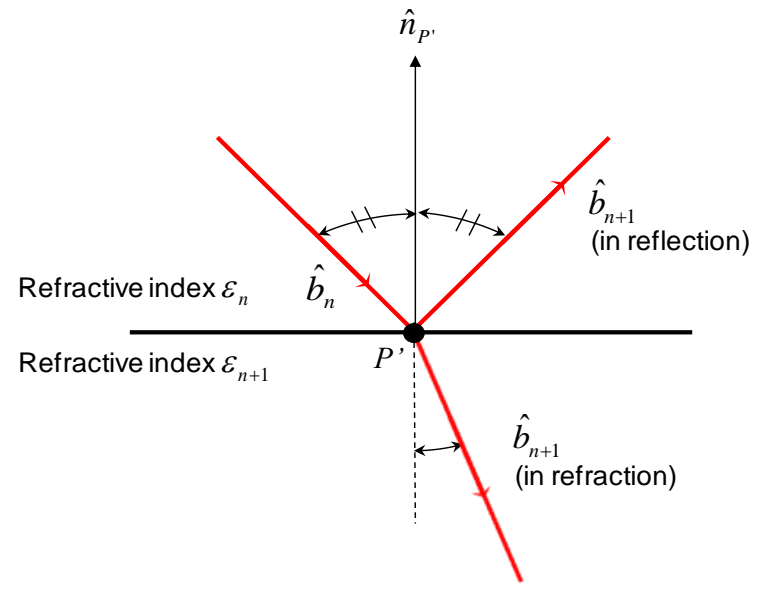

Figure 1 


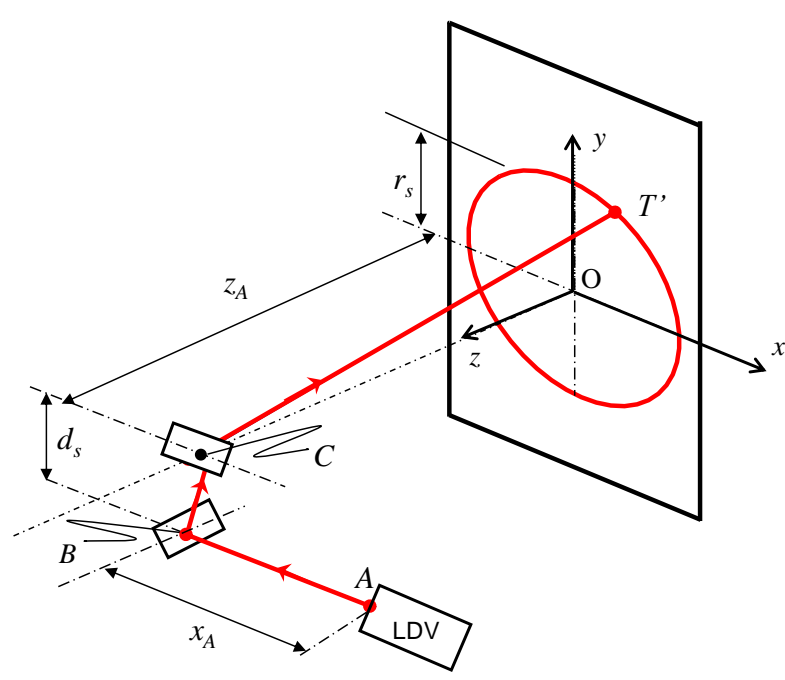

a)

Figure 2

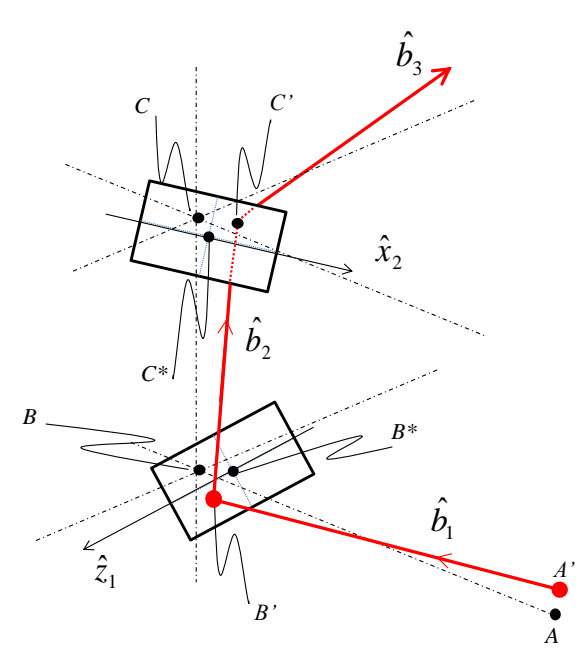

b) 


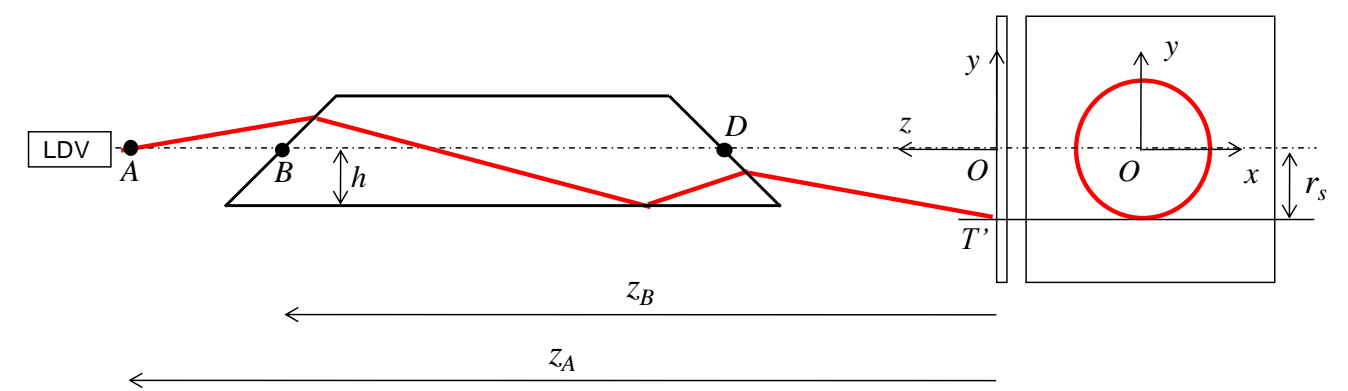

a)

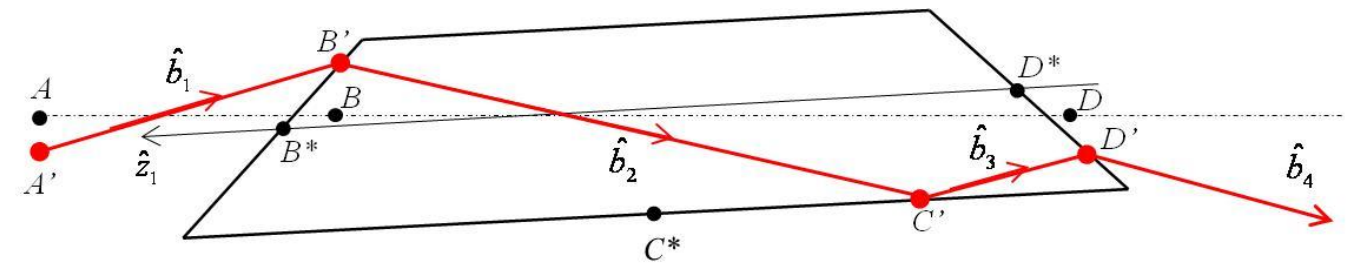

b)

Figure 3 


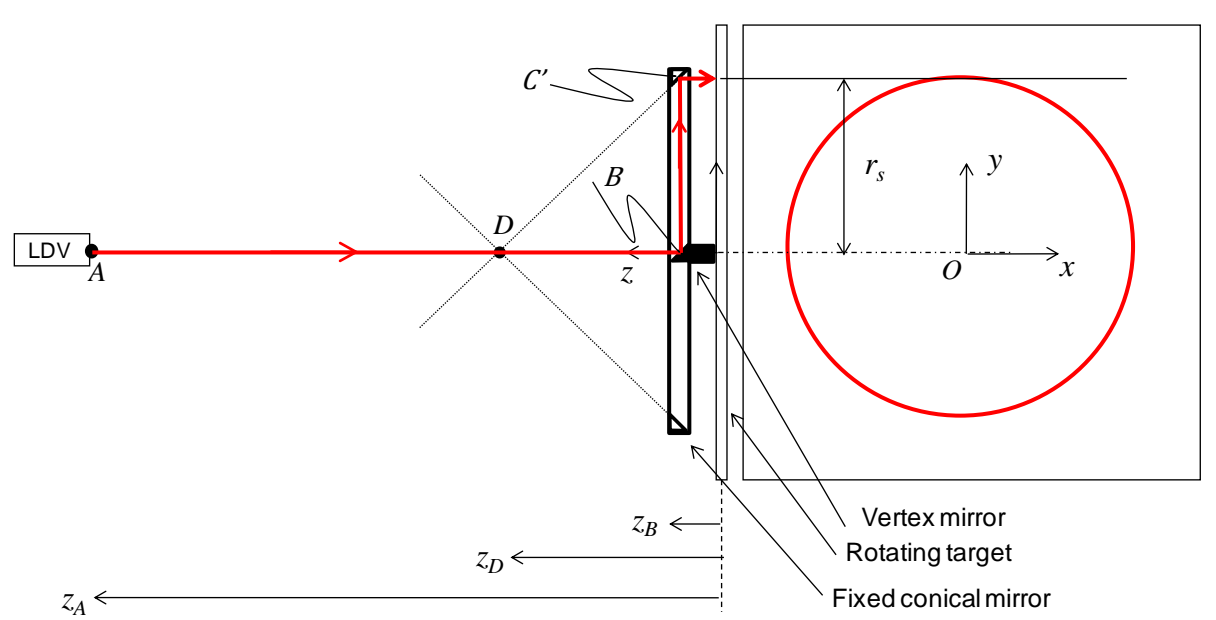

a)

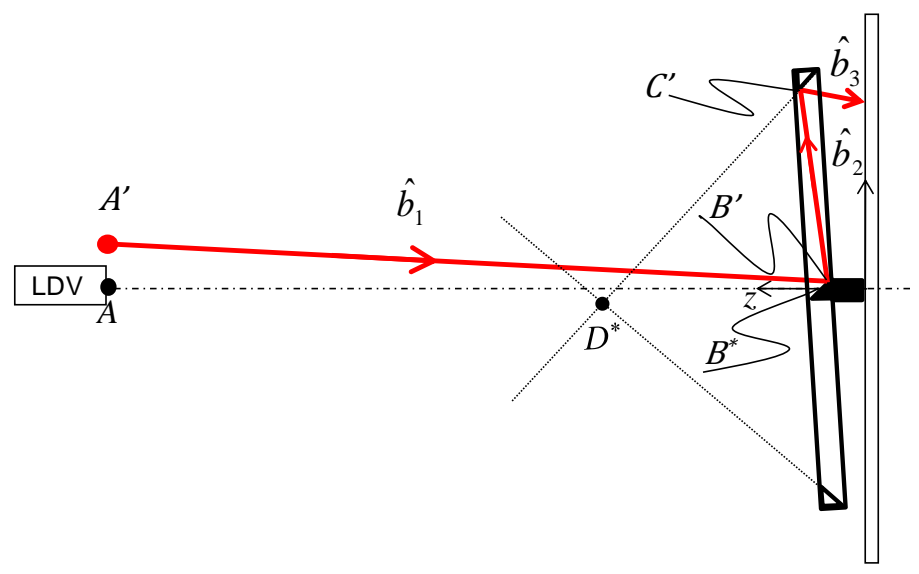

b)

Figure 4 
a)
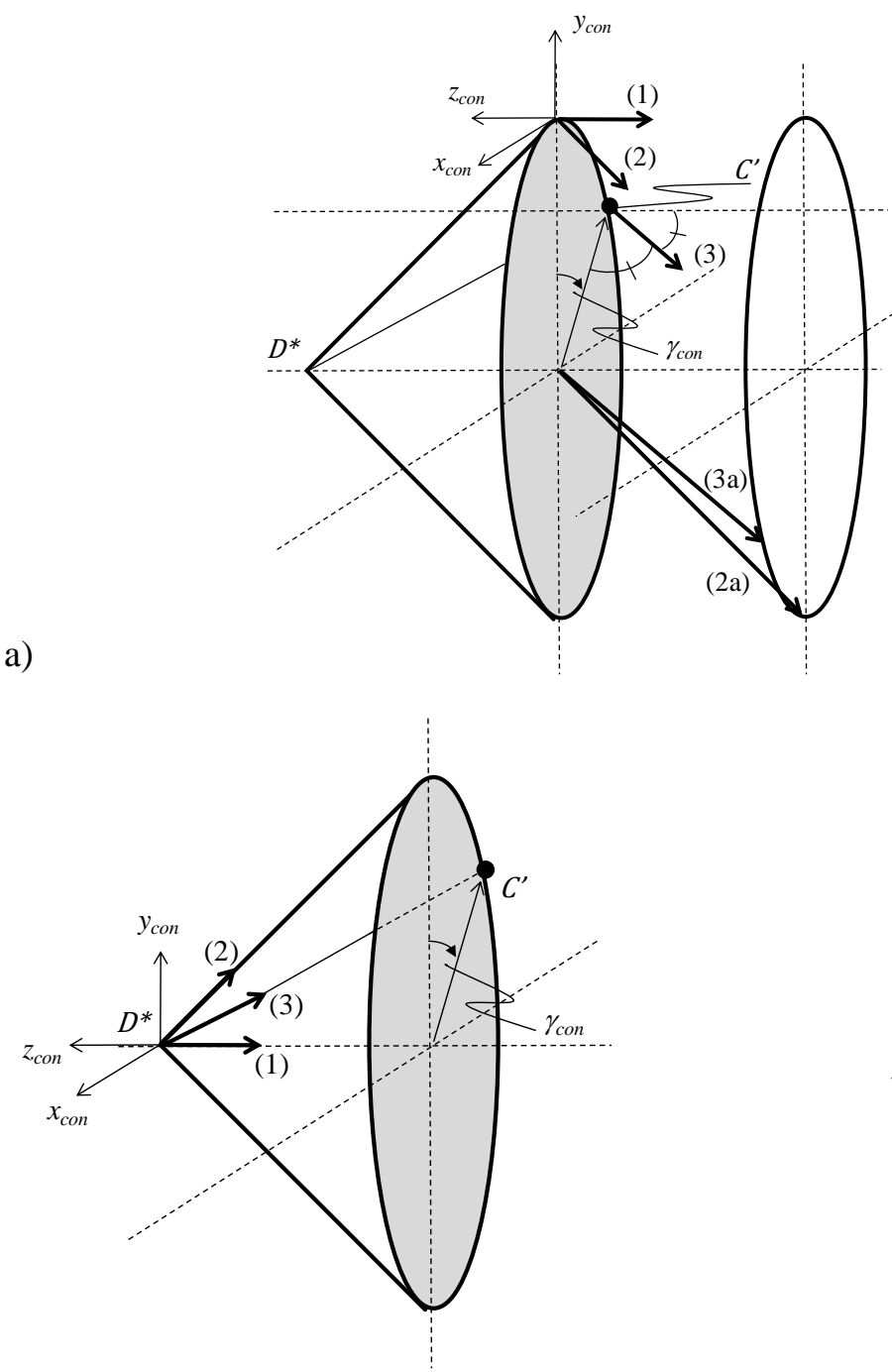

b)

Figure 5

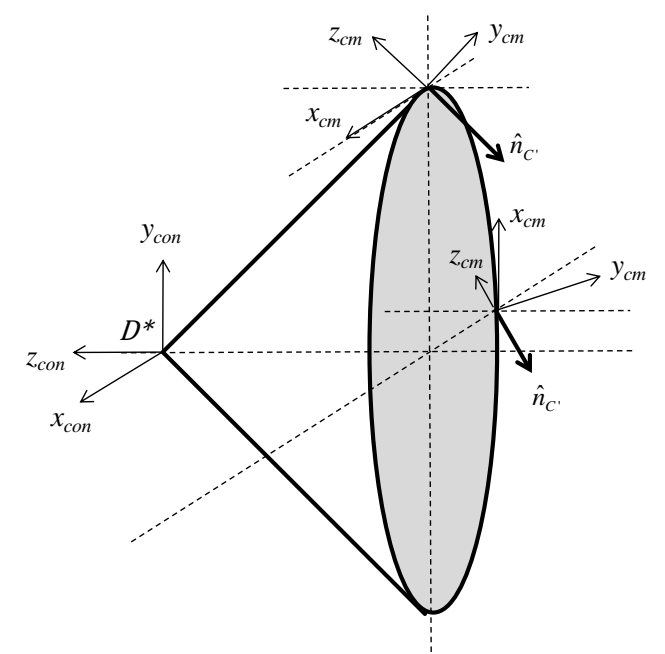

c) 

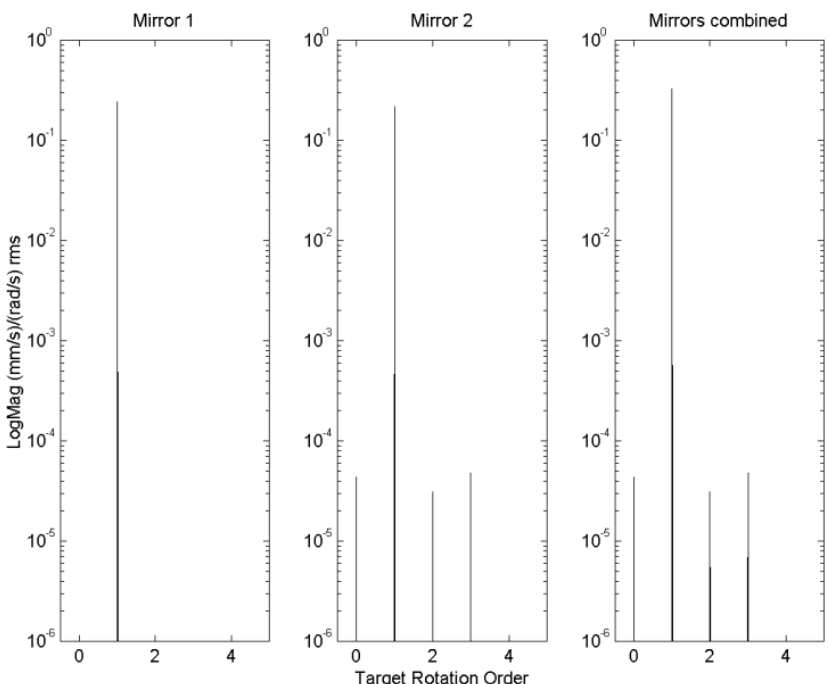

a)
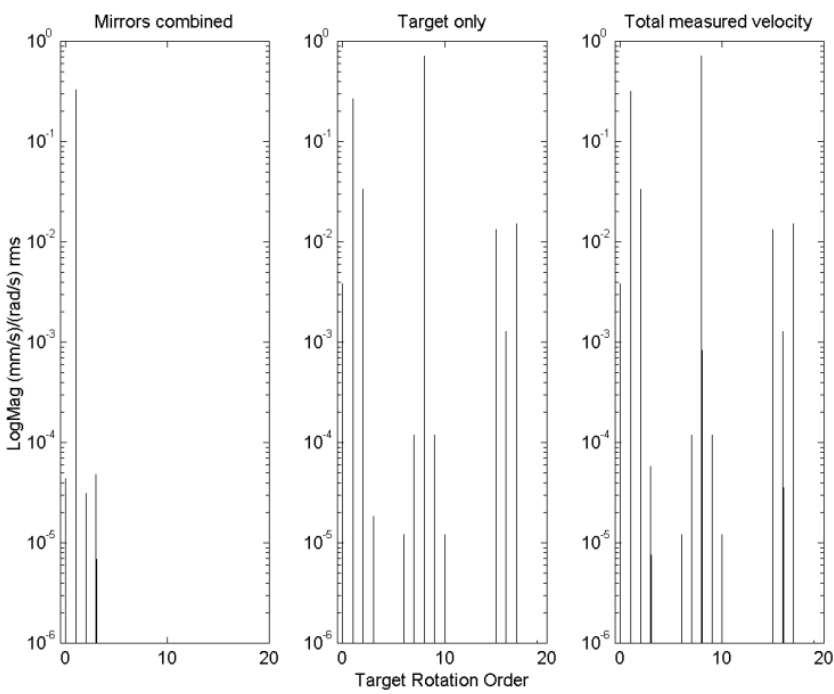

b)

Figure 6 


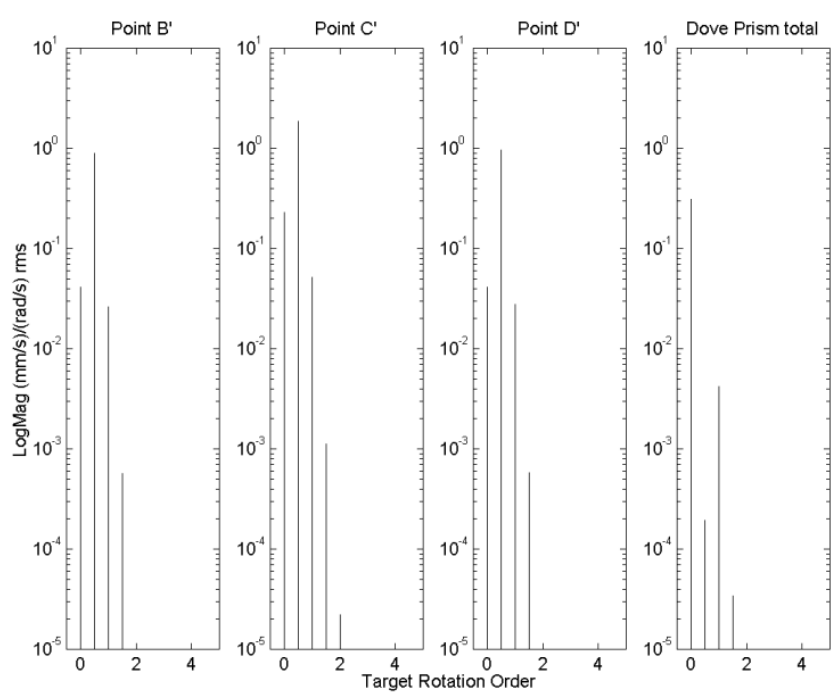

a)
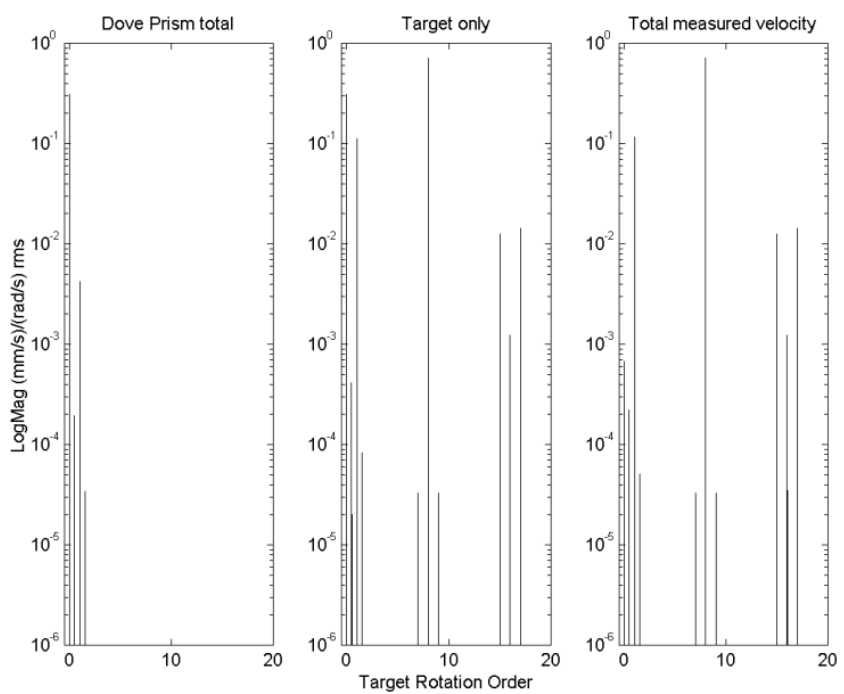

b)

Figure 7 

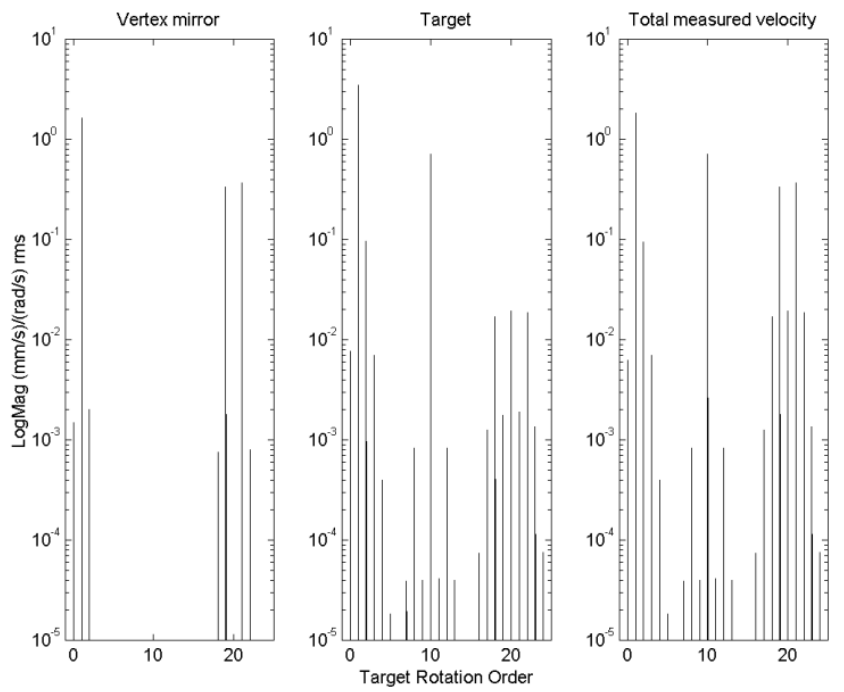

a)
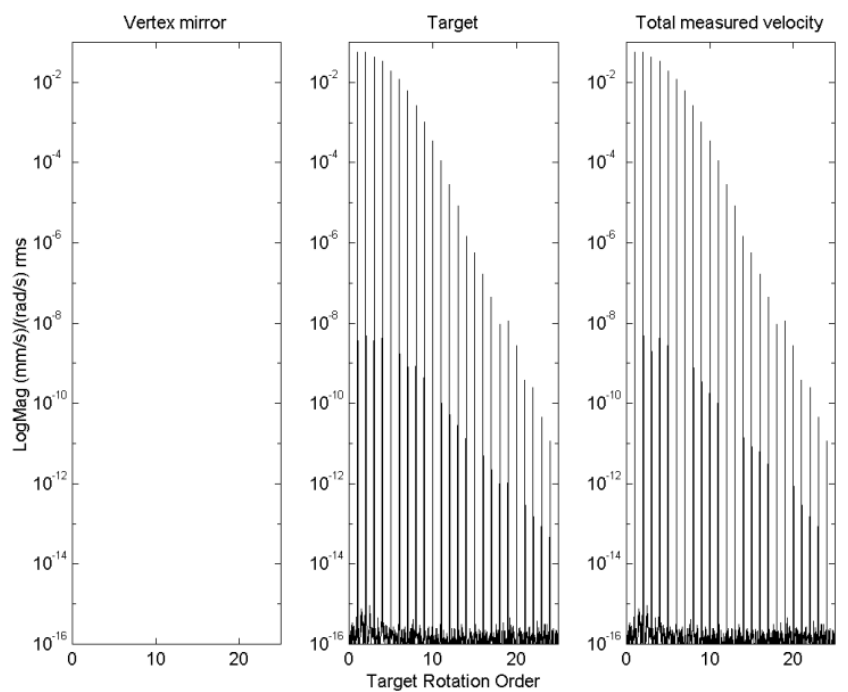

b)
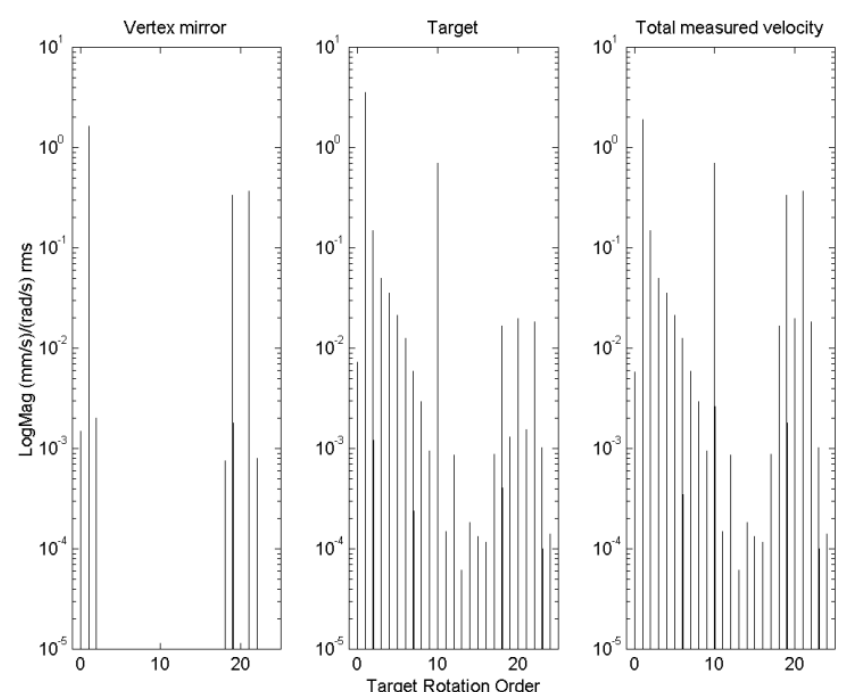

c)

Figure 8 


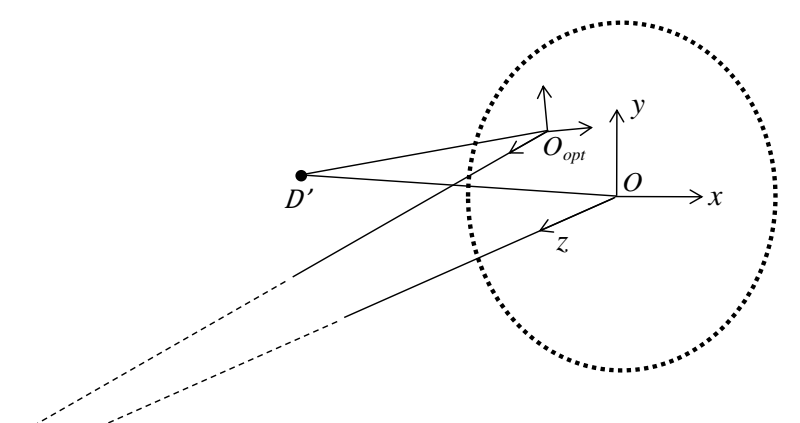

Figure B1 